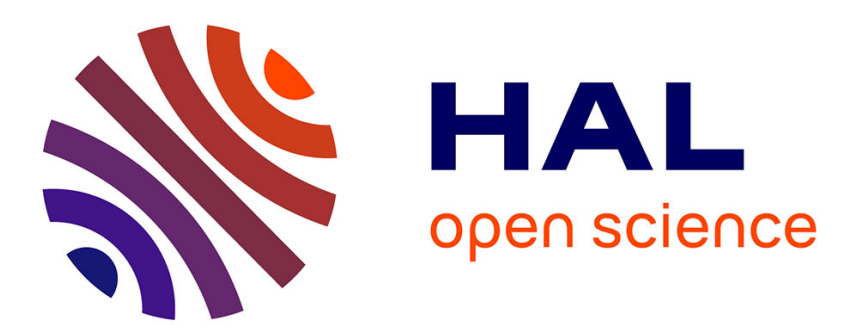

\title{
Non-Decoupled Locomotion and Manipulation Planning for Low-Dimensional Systems
}

Karim Bouyarmane, Abderrahmane Kheddar

\section{To cite this version:}

Karim Bouyarmane, Abderrahmane Kheddar. Non-Decoupled Locomotion and Manipulation Planning for Low-Dimensional Systems. Journal of Intelligent and Robotic Systems, 2018, 91 (3-4), pp.377401. 10.1007/s10846-017-0692-5 . hal-01523752v2

\section{HAL Id: hal-01523752 \\ https://hal.science/hal-01523752v2}

Submitted on 5 Dec 2017

HAL is a multi-disciplinary open access archive for the deposit and dissemination of scientific research documents, whether they are published or not. The documents may come from teaching and research institutions in France or abroad, or from public or private research centers.
L'archive ouverte pluridisciplinaire HAL, est destinée au dépôt et à la diffusion de documents scientifiques de niveau recherche, publiés ou non, émanant des établissements d'enseignement et de recherche français ou étrangers, des laboratoires publics ou privés. 


\title{
Non-Decoupled Locomotion and Manipulation Planning for Low-Dimensional Systems
}

\author{
Karim Bouyarmane · Abderrahmane \\ Kheddar
}

Received: date / Accepted: date

\begin{abstract}
We demonstrate the possibility of solving planning problems by interleaving locomotion and manipulation in a non-decoupled way. We choose three lowdimensional minimalistic robotic systems and use them to illustrate our paradigm: a basic one-legged locomotor, a two-link manipulator with a manipulated object, and a simultaneous locomotion-and-manipulation system. Using existing motion planning and control methods initially designed for either locomotion or manipulation tasks, we see how they apply to both our locomotion-only and manipulationonly systems through parallel derivations, and extend them to the simultaneous locomotion-and-manipulation system. Motion planning is solved for these three systems using two different methods : (i) a geometric path-planning-based one, and (ii) a kinematic control-theoretic-based one. Motion control is then derived by dynamically realizing the geometric paths or kinematic trajectories under the Couloumb friction model using torques as control inputs. All three methods apply successfully to all three systems, showing that the non-decoupled planning is possible.
\end{abstract}

Keywords Locomotion Planning · Manipulation Planning · Contact Planning

\section{Introduction}

Robots are traditionally categorized into fixed-base manipulators $[38,16]$ and mobile navigation robots (wheeled [34] or legged [27]). Many of them, however, do not fall strictly into one of these two categories, as they feature both locomotion and manipulation capabilities and are designed for performing indifferently both

K. Bouyarmane

Université de Lorraine, CNRS, Inria Nancy-Grand Est, Loria UMR 7503, Larsen team, Vandoeuvre-les-Nancy, France.

A. Kheddar

CNRS-AIST Joint Robotics Laboratory (JRL), UMI3218/RL, Tsukuba, Japan.

CNRS-University of Montpellier, LIRMM - Interactive Digital Human group, UMR5506, Montpellier, France. 
kinds of tasks, falling thus into a third locomotion-and-manipulation category. Humanoid robots [30], which constitute the initial motivation that inspired this work, are typical examples of such locomotion-and-manipulation integrated systems.

It is well known that, from a motion planning and control point-of-view, locomotion and manipulation are conceptually the same problems. Their commonality comes from their inherent under-actuation that is solved through the contact forces: a locomotion system is under-actuated in the sense that the position of the mobile base is not controlled directly through actuators torques, but rather results from both the actuation torques action and the contact forces with the support environment; a manipulation system (by manipulation system we mean both the manipulator and the manipulated object) is also under-actuated in a strictly equivalent way: the degrees of freedom of the manipulated object are not actuated and its position is an indirect result of the actuation of the manipulator through the contact forces that it establishes with the manipulated object. Besides, they both obey Lagrangian dynamics, they both involve friction, and they both have contact strata of various dimensions.

Though being equivalent, these two problems have usually been tackled in a decoupled way for integrated manipulation-and-locomotion systems. A decoupled approach might be pertinent for classes of systems in which the initial design imposes totally unrelated locomotion and manipulation components. In that case the decoupled strategy is arguably the most adequate one. However, for systems such as humanoid robots, the frontier between the two kinds of tasks is more blurred, and it is restrictive to exclusively assign upper-body limbs to manipulation and lower-body limbs to locomotion. For instance, a humanoid robot might be required to use its arms to climb a ladder [44] or to crawl under a table [19], it might also need to use its legs to push an object on the floor while walking. In such situations, decoupled approaches using an upper-body joint-space or task-space controller for manipulation and an independent lower-body walking subsystem controller for locomotion [24] can be restrictive and not use the full potential of the human-inspired design.

As for related work, $[31,3,6,5]$ present examples of mechanical designs of robots integrating locomotion and manipulation, other than humanoid robots. Very few works, however, address their motion planning and control problems in an integrated way. [46] considers for example a mobile manipulator and understands the coordinated locomotion and manipulation in the sense of finding the best location of the mobile manipulator to realize the manipulation task. The sequential and functional decoupling of the locomotion and manipulation components is still however existing in this approach, which we aim to erase in ours. A similar remark can be done for approaches such as $[28,29,17,39]$ for humanoid robots, e.g. deploying a virtual mechanism for the footstep placement to find the best fixed footstep location from which the whole-body reaching can be performed. On another level, using a common planning and control framework for locomotion and manipulation is presented in works such as $[49,2]$, but not with a common ground specification of the task letting the planner autonomously decompose it in its locomotion and manipulation components, as necessary for the task completion and taking into account the kinematics and dynamics of the robot.

Our driving objective is to erase high-level distinction between manipulation and locomotion, both in terms of specification of the tasks and of the planning method to plan the motion to realise them. In the resulting motion, interleaved 
manipulation and locomotion should emerge with no prior high-level distinctive formulation. See Fig. 1.

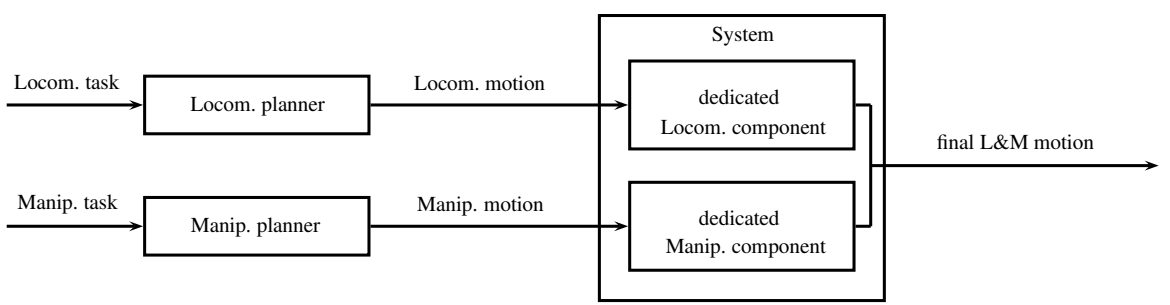

(a) The existing decoupled approach.

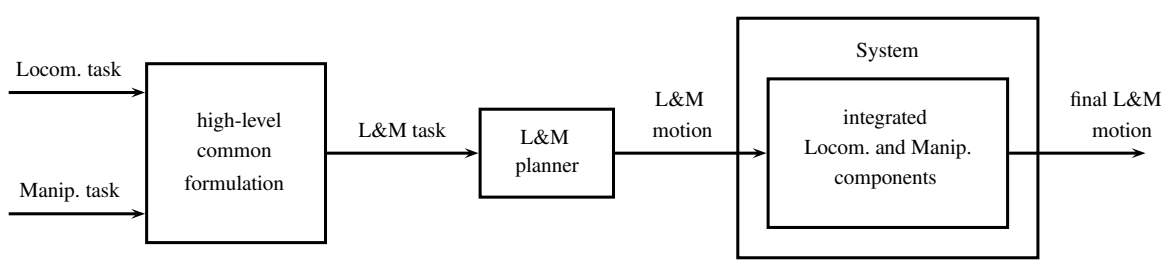

(b) The proposed non-decoupled approach. The L\&M abbreviation stands for Locomotionand-Manipulation

Fig. 1 Overview of the approaches.

The methodology chosen is to capture the locomotion and manipulation problems into the the lowest possible configuration spaces' dimension. The systems are only theoretical planar academic examples but we believe they are still pertinent enough to illustrate our point. As such, this paper is primarily focused on this theoretic and conceptual level and the extension to the above-mentioned humanoid problems is beyond its scope. The three systems we chose are representative of the three categories of robots we mentioned earlier:

- one exclusively locomotion-oriented system,

- one exclusively manipulation-oriented system,

- one hybrid locomotion-and-manipulation (L\&M) system.

We then investigate two main existing motion planning methods from the literature applicable to our systems:

- a geometric path planning approach based on a reduction property proved initially in [1] and used in a randomized planning algorithm in [41];

- a control-theoretic BVP (Boundary Value Problem) approach for kinematic systems based on a controllability theorem proved in [21] and a BVP resolution algorithm developed in [22].

The first approach deals directly with the obstacle avoidance problem. The second is more adequate for dealing with the velocity constraints and nonholonomy which may not translate directly into geometric terms. To make our study complete and self-contained, we also tackle the dynamic trajectory generation problem along the geometric paths resulting from these motion planning algorithms, using the works 
of [42] and [7] as a basis. We derive a time-optimal open-loop torques control law that realizes a given contact motion.

Taking each one of these three motion planning and control techniques, we first apply it the locomotion system, then we show formal equivalence with the manipulation system, before finally extending it to the locomotion-and-manipulation system, which is the main contribution of this work.

Following this methodology, the rest of the paper is structured as follows: Section 2 introduces the three robots we study with their configuration spaces, Section 3 applies the geometric path planning approach to our motion planning problem, Section 4 uses control theory for solving the motion planning problem seen as a BVP, finally Section 5 synthesizes time-optimal control law that realizes the geometric paths provided in previous sections. Each of these sections is divided into three subsections: one for the locomotion robot, one for the manipulation robot, and one for the locomotion-and-manipulation robot.

\section{Systems}

Throughout this paper, we thoroughly study three low-dimensional planar mechanical systems:

$-R_{1}:$ a locomotion robot

$-R_{2}$ : a manipulation robot

- $R_{3}$ : a locomotion-and-manipulation (L \& M) robot

We have chosen these robots for they have the lowest-dimensional possible configuration spaces but yet can capture higher dimensional locomotion and manipulation related concepts. This low dimensionality allows visualizing the configuration spaces in 3D at the expense of simple projections and homeomorphisms. The other purpose of these low-dimensional planar systems is to have explicit analytical expressions for our problems and their solutions.

For all these systems, $\mathcal{C}$ denotes the configuration space, also known as the "Cspace". A configuration is denoted $q \in \mathcal{C}, q$ is the generalized coordinates vector of the system [38]. An important mathematical property in our study is the fact that $\mathcal{C}$ is a smooth manifold. This makes it suited for being described inside the framework of differential geometry theory [26]. Velocities $\dot{q}$ are as such elements of the tangent spaces and generalized forces are elements of the cotangent spaces.

We can classify all the possible forms that the C-space can take for systems commonly considered in robotics. A free-flyer yields the manifold $S E(3)=$ $S O(3) \rtimes \mathbb{R}^{3}$ (semi-direct product). Let $\mathbb{S}^{n}$ be the $n$-dimensional sphere. A revolute joint yields the manifold $\mathbb{S}^{1}$, a spherical joint yields $\mathbb{S}^{3}$. Let $\mathbb{T}^{n}=\left(\mathbb{S}^{1}\right)^{n}$ be the $n$-dimensional torus. A prismatic joint yields the manifold $\mathbb{R}$. In most robotics systems the configuration space $\mathcal{C}$ is a Cartesian product of a given number of these elementary smooth manifolds, thus it is a smooth manifold.

Let $O$ be the obstacle region in the Euclidean workspace. $O$ is a compact subset of $\mathbb{R}^{2}$. Let $\mathcal{C}_{\text {obs }}$ be the image of $O$ in the configuration space, consisting of all configurations where the robot collides with $O \cdot \mathcal{C}_{\text {obs }}$ is a compact subset of $\mathcal{C}$ [33]. Let $\mathcal{C}_{\text {free }}$ be the subspace of $\mathcal{C}$ consisting of all configurations that are not in collision with obstacles, within the joint limits, and not in self-collision. $\mathcal{C}_{\text {free }}$ is an open subset of $\mathcal{C}$ [33]. Studies such as [4] are concerned with the computation 
of explicit representation of the frontier of $\mathcal{C}_{\text {obs }}$ in particular cases, for instance polygonal robots and obstacles in planar world.

We now detail the models and notations for each of the three robotic systems.

\subsection{Locomotion robot}

The robot $R_{1}$ is made of a sliding base along the $x$-axis and a two-link planar manipulator linked with two revolute joints, see Fig. 2. Two actuators control the two revolute joints; the sliding joint is passive, i.e. not actuated and frictionless. The sliding of the base along the $x$-axis can be performed by using friction of end-effector's rubber on the ground.

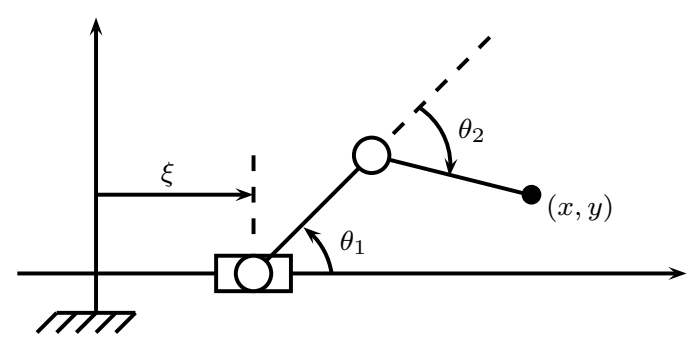

Fig. $2 R_{1}$ and its configuration variables. Rectangles symbolize a prismatic joint while circles represent a revolute joint.

The configuration space of the system is

$$
\mathcal{C}=\mathbb{R} \times \mathbb{T}^{2}
$$

On the manifold $\mathcal{C}$ we use the following coordinates chart $\left(\xi, \theta_{1}, \theta_{2}\right) \in \mathbb{R}^{3}$. We denote by $(x(q), y(q))$ the end effector coordinates in the $(x, y)$-plane. We do not take into account self-collision of the robot. There are no joints limits. $l$ denotes the length of the two links. The robot is not allowed to traverse the ground, thus:

$$
\mathcal{C}_{\text {free }}=\mathcal{C} \backslash \mathcal{C}_{\text {obs }}=\left\{q \in \mathcal{C} \mid \pi>\theta_{1}>0 \text { and } \pi>2 \theta_{1}+\theta_{2}>0\right\}
$$

The 2-dimensional torus $\mathbb{T}^{2}$ is naturally embedded in $\mathbb{R}^{3}$, which means that $\mathcal{C}$ is embedded in $\mathbb{R}^{4}$, thus $\mathcal{C}_{\text {free }}$ is also embedded in $\mathbb{R}^{4}$. However, a projection trick makes it embedded in $\mathbb{R}^{3}$. We simply notice that the projection of $\mathcal{C}_{\text {free }}$ onto $\mathbb{T}^{2}$ is a $2 \mathrm{D}$ manifold which is homeomorphic to a subspace of $(0, \pi) \times \mathbb{S}^{1}$ and thus $\mathcal{C}_{\text {free }}$ is homeomorphic to a subspace of $\mathbb{R} \times(0, \pi) \times \mathbb{S}^{1}$ which is naturally embedded in $\mathbb{R}^{3}$. See Fig. 3 .

The 3D representation of the C-space of $R_{1}$ allows for an explicit representation of $\mathcal{C}_{\text {obs }}$ for any obstacle $O$ for which the frontier is a parameterized 2D curve $\left.\partial O: \rho \mapsto\left(x_{O}(\rho), y_{O}(\rho)\right)\right)$. This parameterized curve would be for example the circle that represents the frontier of the circular obstacle in Fig. 11a.

First let us consider a point obstacle $O$ located at the $\left(x_{O}, y_{O}\right)$ coordinates. The configurations $q$ that make the robot in collision with $O$ can be computed by giving the inverse kinematics solution for the end-effector of a copy robot of $R_{1}$, 

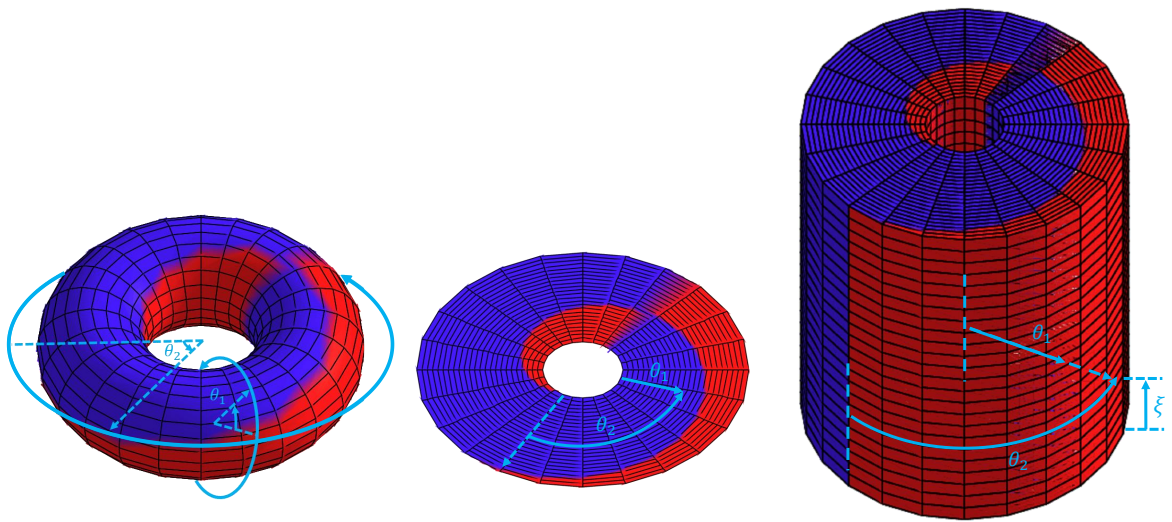

Fig. 3 Embedding the C-space in $\mathbb{R}^{3}$. The blue part represents $\mathcal{C}_{\text {free }}$, the red part is $\mathcal{C} \backslash \mathcal{C}_{\text {free }}$. Left: $\mathbb{T}^{\not \not}$ embedded in $\mathbb{R}^{3}$. Middle: Free part of $\mathbb{T}^{\not \nvdash}$ embedded in $\mathbb{R}^{2}$. Right: Adding the $\xi$ dimension.

but with the second link having a parameter length $\lambda$. Then we make $\lambda$ vary in $[0, l]$, and we get all the configurations $q$ that make the second link of the robot collide with $O$. We use the same method by removing the second link, vary the length of the first link, and compute inverse kinematics for this robot, which gives us the second component of $\mathcal{C}_{\text {obs. }}$. See Fig. 4 .

Now for the full obstacle represented by a parameterized curve $\partial O: \rho \mapsto$ $\left.\left(x_{O}(\rho), y_{O}(\rho)\right)\right)$ we apply the method we have just described by varying the parameter $\rho$.

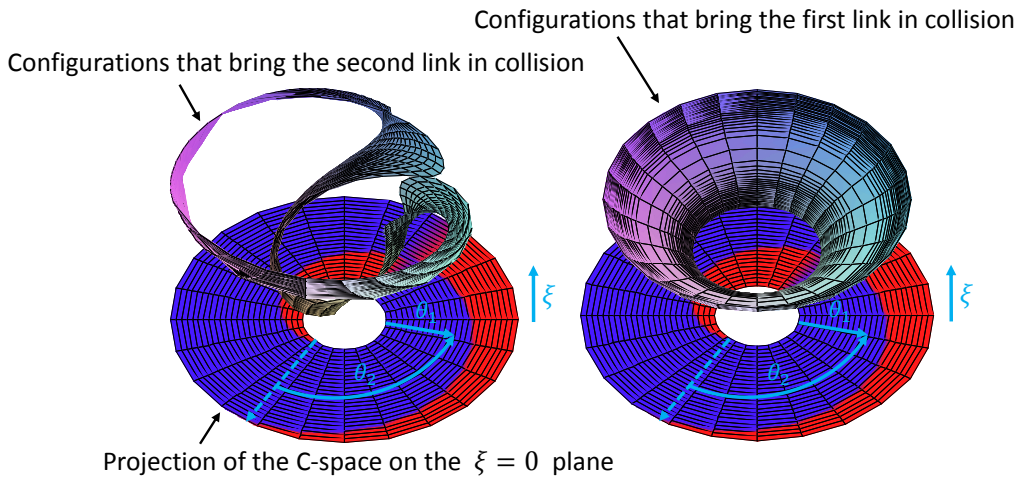

Fig. 4 Components of $\mathcal{C}_{\text {obs }}$ for a point obstacle for the robot $R_{1}$. 


\subsection{Manipulation robot}

The robot $R_{2}$ is a standard two-link planar manipulator fixed to the ground, manipulating a sliding object. See Fig. 5. The manipulated object is pictured in red; it consists of a theoretically infinitely long sliding platform. The manipulator has to put its rubber end-effector on the platform and use friction force to push or pull the object.

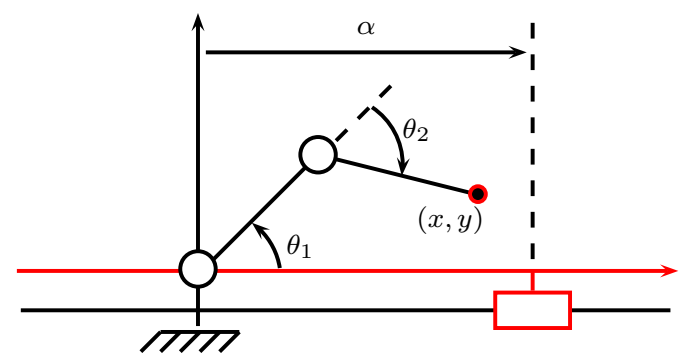

Fig. $5 R_{2}$ and its configuration variables. Joints symbols are the same as Fig. 2. In red the infinitely long sliding platform.

The configuration space of $R_{2}$ is the same as $R_{1}$

$$
\mathcal{C}=\mathbb{R} \times \mathbb{T}^{2}
$$

However we use a different notation for the coordinates chart $\left(\alpha, \theta_{1}, \theta_{2}\right)$ where $\alpha$ denotes the horizontal position of any reference point on the red sliding base. Similarly to $R_{1}$, we consider no self-collision, no joint limits, and Fig. 3 provides a $3 \mathrm{D}$ visualization of $R_{2}$ 's C-space (in the caption read "adding the $\alpha$ dimension" instead of "adding the $\xi$ dimension"). The only difference with $R_{1}$ is the representation of the obstacle region in the C-space, which is basically the $\mathcal{C}_{\text {obs }}$ of a standard two-link manipulator, as detailed in the following paragraph.

To get a parametric representation of $\mathcal{C}_{\text {obs }}$ we use the same trick that we introduced in the computation of $R_{1}$ 's $\mathcal{C}_{\text {obs }}$. For a point obstacle $\left(x_{O}, y_{O}\right)$ we compute the inverse kinematics solution of a robot similar to $R_{2}$ but varying the length of the second link as a parameter $\lambda \in[0, l]$, then we extrude in the $\alpha$ dimension (given that the obstacle region does not depend on the position of the sliding base), we thus get a first component of $\mathcal{C}_{\text {obs }}$ as a $2 \mathrm{D}$ submanifold of $\mathcal{C}$. The second component comes simply from removing the second link and computing the trivial inverse kinematics of a one-link robot, which reduces to a constant $\theta_{1}$. See Fig. 6.

For an obstacle given by a parameterization of its contour $\rho \mapsto\left(x_{O}(\rho), y_{O}(\rho)\right)$, we directly add $\rho$ as a third parameter of our manifold, and we get the representation depicted in Fig. 7 for a circular obstacle for example.

\section{$2.3 \mathrm{~L} \& \mathrm{M}$ robot}

The robot $R_{3}$ combines $R_{1}$ and $R_{2}$. It is made of sliding two-link planar manipulator manipulating an infinitely long sliding platform. See Fig. 8. Its configuration 


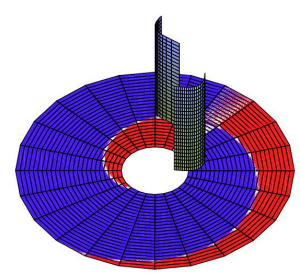

(a) $\mathcal{C}_{\text {obs }, 1}$, corresponding to the configurations that bring the second link into collision with the point.

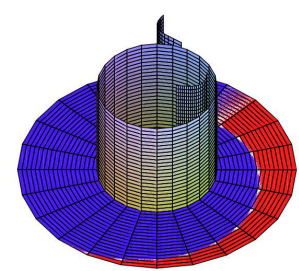

(b) $\mathcal{C}_{\text {obs }, 2}$, corresponding to the configurations that bring the first link into collision with the point.

Fig. 6 Components of $\mathcal{C}_{\text {obs }}$ for a point obstacle for the system $R_{2}$.

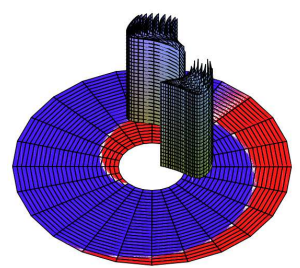

(a) $\mathcal{C}_{\text {obs }, 1}$, corresponding to the configurations that bring the second link into collision with the circle.

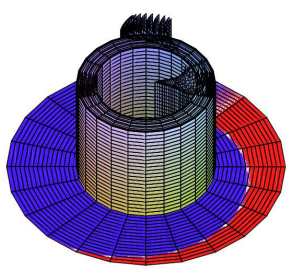

(b) $\mathcal{C}_{\text {obs }, 2}$, corresponding to the configurations that bring the first link into collision with the circle.

Fig. 7 Components of $\mathcal{C}_{\text {obs }}$ for a circular obstacle for the system $R_{2}$.

space is

$$
\mathcal{C}=\mathbb{R}^{2} \times \mathbb{T}^{2}
$$

It is a four-dimensional smooth manifold that cannot be embedded in $\mathbb{R}^{3}$, this time. We skip the representation of the C-space and its obstacle region but we come back to this issue later (Section 3) as we restrain to a special 3D submanifold of the C-space.

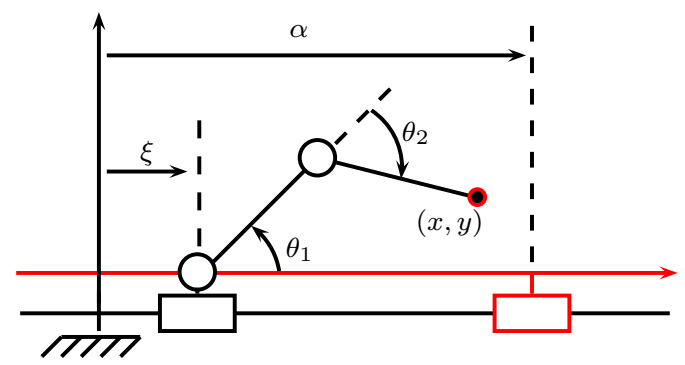

Fig. $8 R_{3}$ and its configuration variables. Rectangles symbolize prismatic joints and circle represent revolute joints. The infinitely long sliding platform is pictured in red. 


\section{Geometric Motion Planning Approach}

The systems introduced in the previous section are underactuated systems. We can geometrically visualize this underactuation as a foliated stratification structure in the C-space.

\subsection{Locomotion robot}

First let us consider the robot $R_{1}$. Its configuration space $\mathbb{R} \times \mathbb{T}^{2}$ is stratified into two different strata, see Fig. 9. The first stratum $\mathcal{S}_{0}$ (zero contact) corresponds to the situation in which the end-effector is not in contact with the ground. It is a submanifold of $\mathcal{C}$ made of all the corresponding configurations

$$
\mathcal{S}_{0}=\left\{\left(\xi, \theta_{1}, \theta_{2}\right) \in \mathbb{R} \times(0, \pi) \times[-\pi, \pi] \mid \theta_{2}>-2 \theta_{1}\right\}
$$

The second stratum $\mathcal{S}_{1}$ (one contact) is the submanifold corresponding to all configurations that bring the end-effector in contact with the ground. It is a 2 dimensional submanifold of $\mathcal{C}$

$$
\mathcal{S}_{1}=\left\{\left(\xi, \theta_{1}, \theta_{2}\right) \in \mathbb{R} \times(0, \pi) \times[-\pi, \pi] \mid \theta_{2}=-2 \theta_{1}\right\}
$$

On this submanifold we use the coordinates chart $\left(\xi, \theta_{1}\right)$

$$
\mathcal{S}_{1}:\left\{\begin{aligned}
\xi & =\xi \\
\theta_{1} & =\theta_{1} \\
\theta_{2} & =-2 \theta_{1}
\end{aligned}\right.
$$

Each of these two strata is foliated into a continuum of leafs. A leaf is a submanifold of the stratum in which the robot is fully actuated. A single leaf of $\mathcal{S}_{0}$ corresponds to a fixed position of the base $\xi$, meaning $\xi=$ constant. We call this foliation the $\xi$-foliation, and for a given $\xi \in \mathbb{R}$ we denote the corresponding leaf $\mathcal{Q}_{0, \xi}$

$$
\mathcal{Q}_{0, \xi}=\left\{\left(\xi, \theta_{1}, \theta_{2}\right) \mid\left(\theta_{1}, \theta_{2}\right) \in(0, \pi) \times[-\pi, \pi] \text { and } \theta_{2}>-2 \theta_{1}\right\}
$$

On $\mathcal{Q}_{0, \xi}, R_{1}$ can move its two links freely in their workspace but does not slide. A single leaf of $\mathcal{S}_{1}$ corresponds to fixed position $x$ of the end-effector on the ground, i.e. $x=$ constant. We call this foliation the $x$-foliation. For a given $x \in \mathbb{R}$ we denote the corresponding leaf $\mathcal{Q}_{1, x}$

$$
\mathcal{Q}_{1, x}=\left\{\left(\xi, \theta_{1}, \theta_{2}\right) \in \mathbb{R} \times(0, \pi) \times[-\pi, \pi] \mid \theta_{2}=-2 \theta_{1} \text { and } \xi+2 l \cos \left(\theta_{1}\right)=x\right\}
$$

or, using the parameter $\theta_{1}$ as coordinate chart,

$$
\mathcal{Q}_{1, x}:\left\{\begin{aligned}
\xi & =x-2 l \cos \left(\theta_{1}\right) \\
\theta_{1} & =\theta_{1} \\
\theta_{2} & =-2 \theta_{1}
\end{aligned}\right.
$$

On such a leaf the robot takes fixed support on the ground and the applied torques result in the sliding of the base.

The purpose of geometric motion planning is to plan a continuous path in the $\mathrm{C}$-space from an initial point to a destination point avoiding the $\mathcal{C}_{\text {obs }}$ region. However in our foliated structure the actuators can only make the robot move 


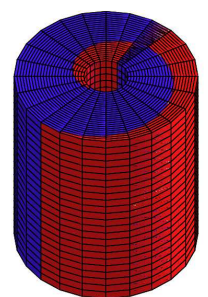

(a) $\mathcal{S}_{0}$ represents the interior of the blue region

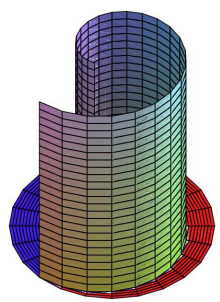

(c) The stratum $\mathcal{S}_{1}$

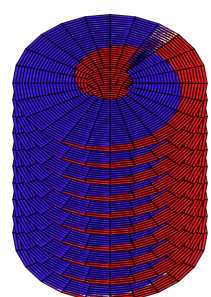

(b) $\xi$-foliation of $\mathcal{S}_{0}$

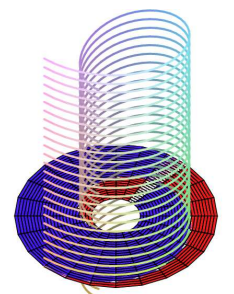

(d) $x$-foliation of $\mathcal{S}_{1}$

Fig. 9 The strata $\mathcal{S}_{0}, \mathcal{S}_{1}$ and their foliations for the robot $R_{1}$.

smoothly along an isolated leaf of the C-space, so the only valid paths should be made of a finite succession of elementary paths along single leafs. This makes the classical techniques of exploring the C-space $[33,15,35]$ not directly applicable to our motion planning problem. However, authors in [41] provide a way to overcome this foliation structure and reduce the problem to a classical motion planning problem in a non-foliated C-space.

In [41], a manipulation path through the C-space is defined as a sequence of transit paths and transfer paths. A transit path is a path in which the object lies at rest on the ground not being manipulated while the manipulator moves freely in its workspace. A transfer path is a path in which the manipulator is grasping the object at a fixed grasp location and the object is "stuck" to the manipulator end-effector. These two kinds of paths are paths along two different strata of the configuration space, respectively the object-stable stratum and the object-grasped stratum. The uncountable infinite stable positions of the object resting on the ground define a foliation of the object-stable stratum, and the uncountable infinite positions of grasps of the end-effector on the object define a foliation on the object-grasped stratum. As shown above, our robot $R_{1}$ fits directly inside this problem formulation. Following the manipulation planning terminology, we call a path through a leaf of $\mathcal{S}_{0}$ a transit path and a path through a leaf of $\mathcal{S}_{1}$ a transfer path. See Fig. 10.

The planning approach developed in [41] is the following: uncover the different connected components of $\mathcal{S}_{1} \cap \operatorname{cl}\left(\mathcal{S}_{0}\right)$ as if there was no foliation structure ${ }^{1}$ (this is done by building a roadmap and connecting the nodes with linear edges thus vio-

\footnotetext{
1 In the remaining of this paper, we denote by $\mathcal{S}_{1} \cap \operatorname{cl}\left(\mathcal{S}_{0}\right)$ the stratum $\mathcal{S}_{1}$ endowed with both the foliation of $\mathcal{S}_{0}$ and the foliation of $\mathcal{S}_{1}$ extended to its topoligical closure, denoted $\operatorname{cl}\left(\mathcal{S}_{1}\right)$.
} 


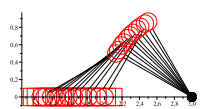

(a) Transfer path in $\mathcal{S}_{1}$

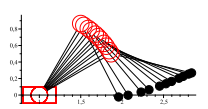

(b) Transit path in $\mathcal{S}_{0}$

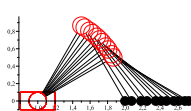

(c) Transit path in $\mathcal{S}_{1} \cap \partial \mathcal{S}_{0}$
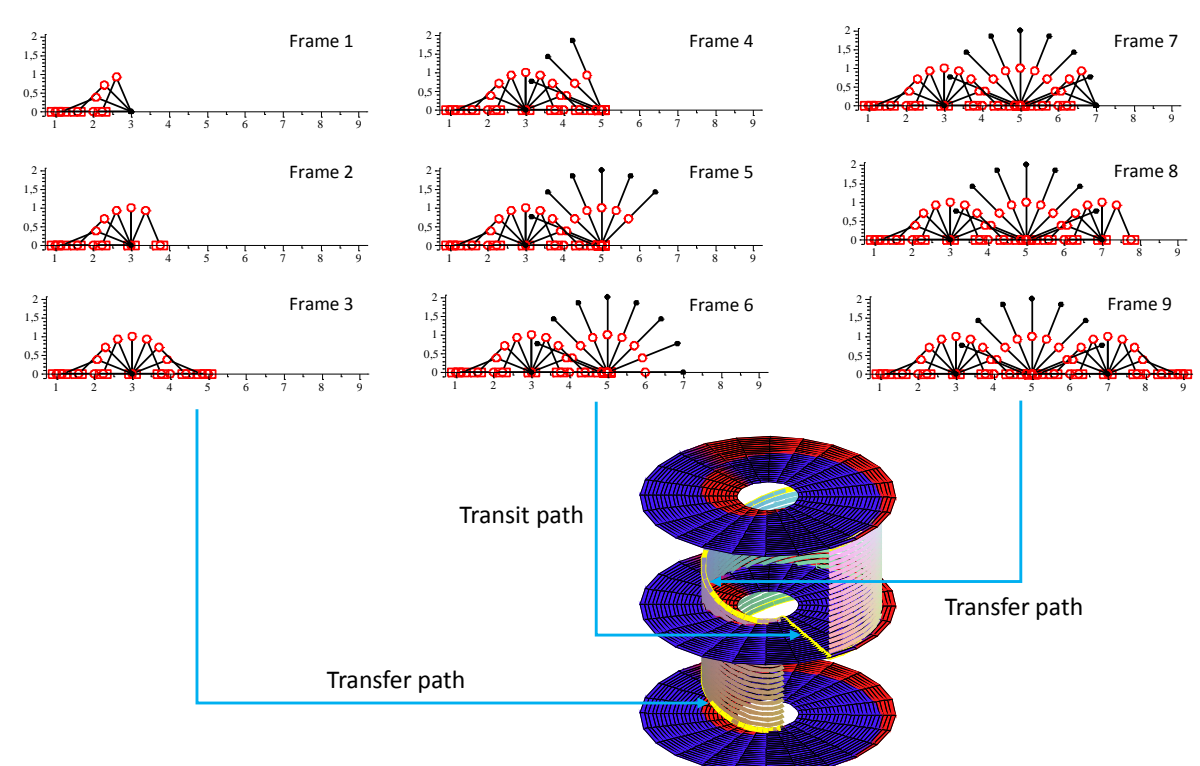

(d) Valid path in the operational space

Fig. 10 Types of paths for the robot $R_{1}$.

lating the foliation structure), then try to connect these different components using only transit or transfer paths. In a post-processing step, The reduction property allows us to transform any collision-free path of $\mathcal{S}_{1} \cap \operatorname{cl}\left(\mathcal{S}_{0}\right)$ into a finite sequence of transfer and transit paths. This reduction property has first been proved in [1]. The following works (e.g. $[41,40])$ based on this property usually assume that the extension of the property is straightforward in their particular problem. However, we believe that the property takes a very specific form in each particular problem and thus needs to be proven on a case-by-case basis, inspired by the general principles of the initial proof. We follow this approach in this section. Moreover, only a constructive proof is candidate to be used as an actual motion planning algorithm. For similar reduction-property-based planning approaches, see [25].

Fig. 11 and Fig. 12 represent the foliation structure on $\mathcal{S}_{0} \cap \mathcal{S}_{1}$. The representation of the obstacle region in Fig. 11 uses the technique presented in section 2. Fig. 12 illustrates the application of the reduction property in a simple case.

Problem 1 Given $\left(q_{\text {initial }}, q_{\text {final }}\right) \in \mathcal{C}_{\text {free }}^{2}$ find $N \in \mathbb{N}$, a sequence $\left(k_{i}\right)_{i=1 \cdots N} \in$ $\{0,1\}^{N}$, a sequence $\left(\zeta_{i}\right)_{i=1 \cdots N} \in \mathbb{R}^{N}$, and a sequence of continuous paths $p_{i}$ : 


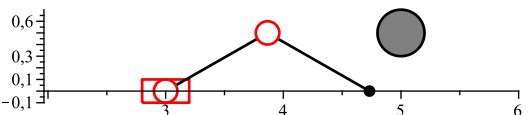

(a) A circular obstacle in the operational space.

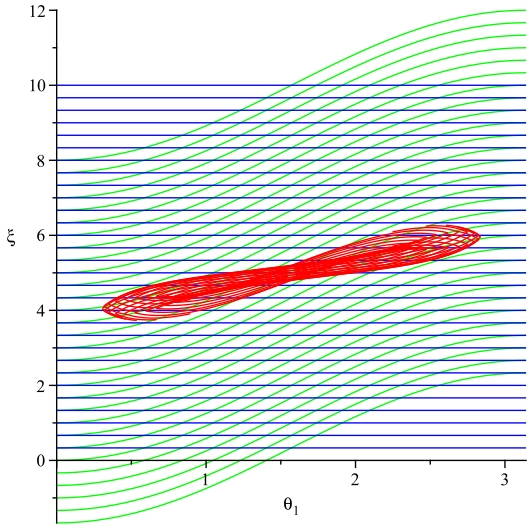

(b) The obstacle region in the foliated $\mathcal{S}_{0} \cap$ $\mathcal{S}_{1}$ in represented in red. The blue foliation is the $\xi$-foliation, the green foliation is the $x$-foliation.

Fig. 11 Example of an obstacle and its mapping in the foliated spaces.

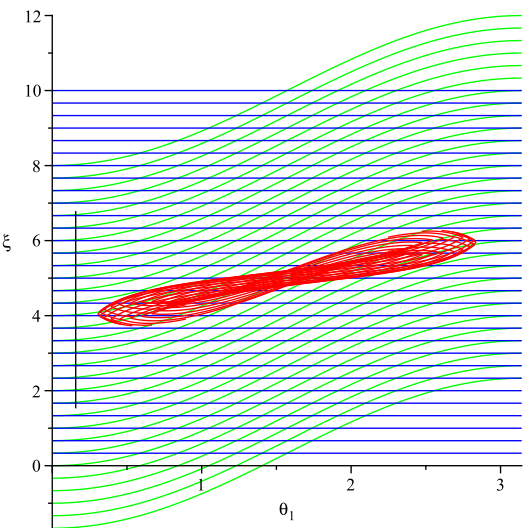

(a) Original path (black vertical path on the left)

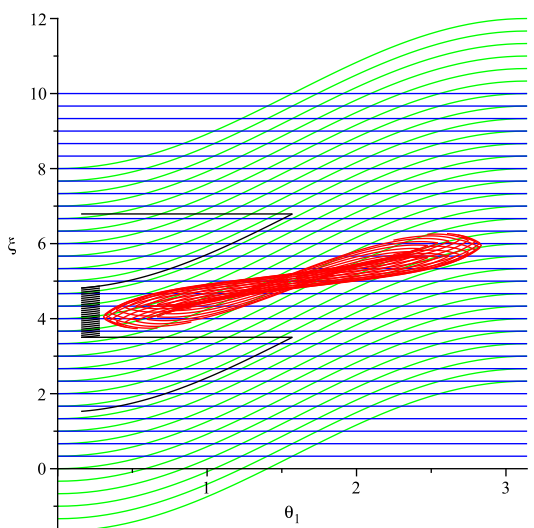

(b) Valid path

Fig. 12 Illustration of the reduction property. In the first figure the black vertical linear path in the left of the figure violates the foliation. In the second figure the path is deformed in order to comply with the foliation.

$[0,1] \rightarrow \mathcal{Q}_{k_{i}, \zeta_{i}} \cap \mathcal{C}_{\text {free }}$, such that $p_{0}(0)=q_{\text {initial }}, p_{N}(1)=q_{\text {final }}$, and $\forall i \in\{0, \ldots, N-$ 1) $p_{i}(1)=p_{i+1}(0)$.

Proposition 1 If there exists for $R_{1}$ a collision-free path in unfoliated $\mathcal{S}_{1} \cap \operatorname{cl}\left(\mathcal{S}_{0}\right)$ from $q_{\text {initial }}$ to $q_{\text {final }}$ then there exists a finite sequence of transfer and transit paths that links $q_{\text {initial }}$ and $q_{\text {final }}$.

Proof The two foliations of $\mathcal{S}_{1} \cap \mathrm{cl}\left(\mathcal{S}_{0}\right)$ can be respectively represented by the two families of functions $\left(f_{\alpha}\right)_{\alpha \in \mathbb{R}}$ and $\left(g_{\beta}\right)_{\beta \in \mathbb{R}}$ (where $\alpha$ and $\beta$ are formally bound 
variables), defined for any real value $\alpha \in \mathbb{R}$ and any real value $\beta \in \mathbb{R}$ as:

$$
\begin{aligned}
f_{\alpha}:(0, \pi) & \rightarrow \mathbb{R} \\
\theta_{1} & \mapsto \xi=f_{\alpha}\left(\theta_{1}\right)=\alpha
\end{aligned}
$$

which represents the horizontal foliation (the $\xi$-foliation) and where $\alpha$ represents the fixed position of the base, and

$$
\begin{aligned}
g_{\beta}:(0, \pi) & \rightarrow \mathbb{R} \\
\theta_{1} & \mapsto \xi=g_{\beta}\left(\theta_{1}\right)=-2 \cos \left(\theta_{1}\right)+\beta
\end{aligned}
$$

which represents the curved inclined foliation (the $x$-foliation) and where $\beta$ represents the fixed position of the contact tip.

For more convenience in the notations we replace the $\left(\theta_{1}, \xi\right)$ coordinate chart notation on $\mathcal{S}_{0} \cap \mathcal{S}_{1}$ by the more usual plane coordinates $(x, y)$. We also denote $\mathcal{C}=(0, \pi) \times \mathbb{R}$ as our ambient metric space, and the obstacle region $\mathcal{O}$ which is a non-empty compact (ie. closed and bounded) subset of $\mathcal{C}$. The complementary set of $\mathcal{O}$ that we denote $\mathcal{O}^{c}=\mathcal{C} \backslash \mathcal{O}$ is an open subset of $\mathcal{C}$. The distance between two subsets $A$ and $B$ of $\mathcal{C}$ is defined as:

$$
d(A, B)=\inf _{a \in A, b \in B} d(a, b)
$$

The two foliations on $\mathcal{C}$ are now represented by the two families of functions: $f_{\alpha}(x)=\alpha, \alpha \in \mathbb{R}$ and $g_{\beta}(x)=g(x)+\beta, \beta \in \mathbb{R}$ where $g:(0, \pi) \rightarrow \mathbb{R}$ is a continuous strictly increasing function.

In our demonstration we first consider the case of an initial vertical path. Let $p_{v}:[0,1] \rightarrow \mathcal{O}^{c}$ be a normal parametrization of our vertical path (arc-length parametrization) from the bottom extremity, ie. $p_{v}(t)=\left(x_{0}, y_{0}+t \cdot l\right)$ where $l$ is the length of the path. Let $\operatorname{Tr}\left(p_{v}\right)=\left\{p_{v}(t) \mid t \in[0,1]\right\}$. Since $\operatorname{Tr}\left(p_{v}\right)$ and $\mathcal{O}$ are two non-empty compact subsets of $\mathcal{C}$, their distance is finite: $d\left(\operatorname{Tr}\left(p_{v}\right), \mathcal{O}\right)<+\infty$. Since they are closed sets with empty intersection $\operatorname{Tr}\left(p_{v}\right) \cap \mathcal{O}=\varnothing$ their distance is strictly positive $d\left(\operatorname{Tr}\left(p_{v}\right), \mathcal{O}\right)>0$. Let $\varepsilon=\frac{d\left(\operatorname{Tr}\left(p_{v}\right), \mathcal{O}\right)}{2}$.

We now give a recursive construction of a finite sequence of collision-free transit paths and transfer paths that links $\left(x_{0}, y_{0}\right)$ to $\left(x_{0}, y_{0}+l\right)$.

From the foliation definition, we know that $\exists \beta_{0} \in \mathbb{R} \mid g_{\beta_{0}}\left(x_{0}\right)=y_{0}$. Let $B_{0}$ be the closed ball of center $\left(x_{0}, y_{0}\right)$ and of radius $\varepsilon$. From the construction of $\varepsilon$ we have $B_{0} \subset \mathcal{O}^{c}$. Let $y=a(x)$ be the equation of the closed upper right quarter circle boundary of $B_{0}$. We have $a\left(x_{0}\right)=g_{\beta_{0}}\left(x_{0}\right)+\varepsilon>g_{\beta_{0}}\left(x_{0}\right)$ and $g_{\beta_{0}}\left(x_{0}+\varepsilon\right)>$ $g_{\beta_{0}}\left(x_{0}\right)=a\left(x_{0}+\varepsilon\right)$. The intermediate value theorem applied to the continuous strictly increasing function $g_{\beta_{0}}-a$ ( $a$ being continuous strictly decreasing function) gives us a unique point $\left(x_{0}^{\prime}, y_{1}\right)$ of intersection between the graphs of $g_{\beta_{0}}$ and $a$ such that $\left(x_{0}^{\prime}, y_{1}\right) \in\left(x_{0}, x_{0}+\varepsilon\right) \times\left(y_{0}, y_{0}+\varepsilon\right)$. Because of $B_{0}$ being strictly convex, the horizontal line segment between the points $\left(x_{0}^{\prime}, y_{1}\right)$ and $\left(x_{0}, y_{1}\right)$ is inside $B_{0}$. Let $\alpha_{0}=y_{1}$. Finally we have constructed a sequence of two paths

$$
\begin{aligned}
\text { Transfer }_{0}:\left[x_{0}, x_{0}^{\prime}\right] & \rightarrow \mathcal{O}^{c} \\
x & \mapsto\left(x, g_{\beta_{0}}(x)\right)
\end{aligned}
$$

and

$$
\begin{aligned}
\text { Transit }_{0}:\left[-x_{0}^{\prime},-x_{0}\right] & \rightarrow \mathcal{O}^{c} \\
x & \mapsto\left(-x, f_{\alpha_{0}}(-x)\right)
\end{aligned}
$$


that link $\left(x_{0}, y_{0}\right)$ to $\left(x_{0}, y_{1}\right)$. Let $d=y_{1}-y_{0} . d>0$ from the above definition of $y_{1}$. Let $N=\left\lfloor\frac{l}{d}\right\rfloor$. Repeating the previous procedure from the point $\left(x_{0}, y_{1}\right)$, we recursively define a sequence of points along $\operatorname{Tr}\left(\mathrm{p}_{\mathrm{v}}\right),\left(x_{0}, y_{n}\right)_{0 \leq n \leq N}$ where $y_{n}=$ $y_{0}+n d$ and the corresponding sequences of paths $\left(\text { Transfer }_{n}, \text { Transit }_{n}\right)_{0 \leq n \leq N-1}$ that link $\left(x_{0}, y_{n}\right)$ to $\left(x_{0}, y_{n+1}\right)$. To end the recursion, Let $y_{N+1}=y_{0}+l$ and $x_{N}^{\prime}=g_{\beta_{N}}^{-1}\left(y_{N+1}\right)$. The last transit and transfer paths of the sequence are defined as:

$$
\begin{aligned}
\text { Transfer }_{\mathrm{N}}:\left[x_{N}, x_{N}^{\prime}\right] & \rightarrow \mathcal{O}^{c} \\
x & \mapsto\left(x, g_{\beta_{N}}(x)\right)
\end{aligned}
$$

and

$$
\begin{aligned}
\operatorname{Transit}_{N}:\left[-x_{N}^{\prime},-x_{N}\right] & \rightarrow \mathcal{O}^{c} \\
x & \mapsto\left(-x, f_{\alpha_{N}}(-x)\right)
\end{aligned}
$$

Finally, the sequence $\left(\operatorname{Transfer}_{n}, \text { Transit }_{n}\right)_{0 \leq n \leq N}$ link the initial and final point of our vertical path $p_{v}$, which ends the first part of the demonstration.

Let us now consider a given non-necessarily vertical path from $\left(x_{0}, y_{0}\right)$ to $\left(x_{f}, y_{f}\right), p:[0,1] \rightarrow \mathcal{O}^{c}$. We suppose that $p$ is a normal (arc-length) parametrization, otherwise we can re-parametrize under the condition that $p$ is regular, meaning that $\forall t \in[0,1], \dot{p}(t) \neq(0,0)$. Let $l$ be the length of the path.

We first show that we can find a finite sequence of collision-free vertical and horizontal paths that link $\left(x_{0}, y_{0}\right)$ to $\left(x_{f}, y_{f}\right)$. Once again we define $\varepsilon=\frac{d(\operatorname{Tr}(p), \mathcal{O})}{2}$. Let $N=\min \left\{n \in \mathbb{N} \mid \frac{l}{n}<\varepsilon\right\}$. We define the sequence of points along $\operatorname{Tr}(p)$, $\left(x_{n}, y_{n}\right)_{0 \leq n \leq N}$ such that $\left(x_{n}, y_{n}\right)=p\left(\frac{n}{N}\right)$, for $0 \leq n \leq N$. Now for each $0 \leq n \leq$ $N-1$, we define the following sequence of horizontal and vertical paths:

$$
\begin{aligned}
\text { Horizontal }_{\mathrm{n}}:\left[x_{n}, x_{n+1}\right] & \rightarrow \mathcal{O}^{c} \\
x & \mapsto\left(x, y_{n}\right)
\end{aligned}
$$

and

$$
\begin{aligned}
\text { Vertical }_{\mathrm{n}}:\left[y_{n}, y_{n+1}\right] & \rightarrow \mathcal{O}^{c} \\
y & \mapsto\left(x_{n+1}, y\right)
\end{aligned}
$$

(the notations of the intervals above depends on the relative ordering of $x_{n}$ and $x_{n+1}$, and of $y_{n}$ and $\left.y_{n+1}\right)$. Note that $\left[\left(x_{n}, y_{n}\right),\left(x_{n+1}, y_{n+1}\right)\right]$ is the hypotenuse of the triangle $\left(x_{n}, y_{n}\right),\left(x_{n+1}, y_{n}\right),\left(x_{n+1}, y_{n+1}\right)$, so the length of the two paths above are less than the length of the chord $\left[\left(x_{n}, y_{n}\right),\left(x_{n+1}, y_{n+1}\right)\right]$, which is less than the arc-length from $\left(x_{n}, y_{n}\right)$ to $\left(x_{n+1}, y_{n+1}\right)$, which is by construction equal to $\frac{l}{N}<\varepsilon$. This means that the two sequences of paths Horizontal $_{n}$ and Vertical $_{n}$ are effectively included in $\mathcal{O}^{c}$, ie. are collision-free.

All in all, we constructed a finite sequence of collision-free vertical and horizontal paths from $q_{\text {initial }}$ to $q_{\text {final }}$. Each horizontal path is already a transit path. Each vertical path can be decomposed using the first part of this demonstration in a finite sequence of transfer and transit paths. This means that we constructed a finite sequence of transfer and transit paths that link $q_{\text {initial }}$ and $q_{\text {final }}$.

\subsection{Manipulation robot}

All the development provided in the previous section for $R_{1}$ is strictly valid for $R_{2}$ modulo some slight changes of referential and notations. The system being a manipulation system, the terminology in [41] applies now directly to $R_{2}$. 
To adapt the development of the previous section from $R_{1}$ to $R_{2}$ we first need to replace all the occurrences of $\xi$ by $\alpha$. For example, we call $\alpha$-foliation instead of $\xi$-foliation for $\mathcal{S}_{0}$. For a fixed $\alpha \in \mathbb{R}$, a leaf $\mathcal{Q}_{0, \alpha}$ of this foliation corresponds to a fixed location of the sliding platform while the manipulator moves freely in its workspace.

For the stratum $\mathcal{S}_{1}$ the foliation should correspond to the different possible locations of the contact point which be fixed in the inertial frame of the sliding platform. So we introduce a new variable $\beta=\alpha-x$ (see Fig. 13a) which becomes the new co-parameter of $\mathcal{S}_{1}$ foliation, that we call the $\beta$-foliation (instead of the $x$-foliation for $R_{1}$ ). See Fig. 14 . For $\beta \in \mathbb{R}$, a leaf $\mathcal{Q}_{1, \beta}$ is written as

$$
\mathcal{Q}_{1, \beta}=\left\{\left(\alpha, \theta_{1}, \theta_{2}\right) \in \mathbb{R} \times(0, \pi) \times[-\pi, \pi] \mid \theta_{2}=-2 \theta_{1} \text { and } 2 l \cos \left(\theta_{1}\right)+\beta=\alpha\right\}
$$

or, using the parameter $\theta_{1}$ as coordinate chart,

$$
\mathcal{Q}_{1, \beta}:\left\{\begin{aligned}
\alpha & =\beta+2 l \cos \left(\theta_{1}\right) \\
\theta_{1} & =\theta_{1} \\
\theta_{2} & =-2 \theta_{1}
\end{aligned}\right.
$$

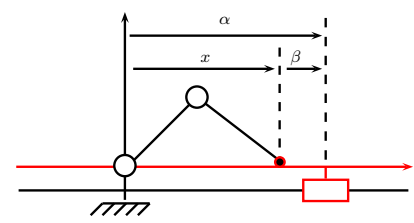

(a) $R_{2}$

Fig. 13 The $\beta$ variable.

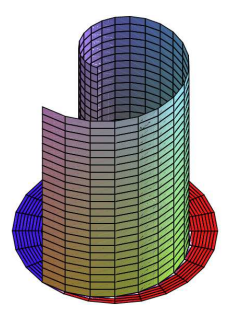

(a) The stratum $\mathcal{S}_{1}$ of $R_{2}$

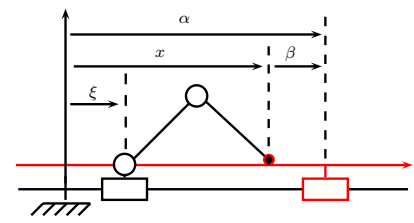

(b) $R_{3}$

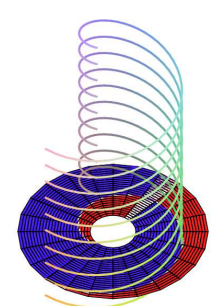

(b) $\beta$-foliation of $\mathcal{S}_{1}$

Fig. 14 The stratum $\mathcal{S}_{1}$ and its foliation for the system $R_{2}$.

Proposition 2 If there exists for $R_{2}$ a collision-free path in unfoliated $\mathcal{S}_{1} \cap \operatorname{cl}\left(\mathcal{S}_{0}\right)$ from $q_{\text {initial }}$ to $q_{\text {final }}$ then there exists a finite sequence of transfer and transit paths that links $q_{\text {initial }}$ and $q_{\text {final }}$. 
Proof For $R_{2}$, the two foliations of $\mathcal{S}_{1} \cap \mathrm{cl}\left(\mathcal{S}_{0}\right)$ can be respectively represented by the two families of functions:

$$
\begin{aligned}
f_{\mu}:(0, \pi) & \rightarrow \mathbb{R} \\
\theta_{1} & \mapsto \alpha=f_{\mu}\left(\theta_{1}\right)=\text { constant }=\mu, \quad \mu \in \mathbb{R}
\end{aligned}
$$

which represents the horizontal foliation (the $\alpha$-foliation), and

$$
\begin{aligned}
g_{\nu}:(0, \pi) & \rightarrow \mathbb{R} \\
\theta_{1} & \mapsto \alpha=g_{\nu}\left(\theta_{1}\right)=2 \cos \left(\theta_{1}\right)+\nu, \quad \nu \in \mathbb{R}
\end{aligned}
$$

which represents the curved inclined foliation (the $\beta$-foliation).

The argument used in the proof of proposition 1 was that the function $g$ is a strictly increasing function which allowed us to apply the intermediate value theorem. Actually, we only need strict monotony to reach the same conclusion. In our present case the corresponding function $g$ is strictly decreasing, so the proof of proposition 1 is valid for proposition 2 .

\section{$3.3 \mathrm{~L} \& \mathrm{M}$ robot}

We now consider the robot $R_{3}$. Similarly to $R_{2}$ we define the variable $\beta=\alpha-x$ as pictured in Fig. 13b.

The configuration space of the robot is 4-dimensional $\mathbb{R}^{2} \times \mathbb{T}^{2}$ parametrized by $\left(\xi, \theta_{1}, \theta_{2}, \alpha\right)$. We still have only two actuators at the revolute joints, therefore the degree of underactuation is $4-2=2$. However, we also still have only one possible contact force to resolve the underactuation and reduce its degree by one. One possible way of resolving the last remaining degree of underactuation is to add a discrete switching control variable $u_{d} \in\{0,1\}$ which allow us to either block the manipulator's base and release the sliding platform (case $u_{d}=0$ ) or release the manipulator's base and block the sliding platform (case $u_{d}=1$ ).

Using the terminology of hybrid control theory, we consider the following discrete "states" of the robot:

- The free mode. The manipulator's base and the sliding platform are fixed, i.e. $\xi=$ constant and $\alpha=$ constant. This defines a first state in which the manipulator's links $\left(\theta_{1}, \theta_{2}\right)$ move freely in their workspace.

- The manipulation mode. The manipulator's base is fixed and the end-effector is in contact with the sliding platform at fixed position in the platform's frame, i.e. $\xi=$ constant and $\beta=$ constant. This defines a second state in which the manipulator pushes or pulls the platform.

- The locomotion mode. The sliding platform is fixed and the end-effector is in contact with the sliding platform at fixed position in the platform's frame, i.e. $\alpha=$ constant and $\beta=$ constant. This defines a last state in which the manipulator pushes or pulls itself.

We still have two strata: $\mathcal{S}_{0}=\mathcal{C}$ and $\mathcal{S}_{1}: \theta_{2}=-2 \theta_{1}$. However, $\mathcal{S}_{1}$ is now a three dimensional submanifold on which we use the coordinate chart $\left(\xi, \alpha, \theta_{1}\right)$. The two states -locomotion and manipulation- are both defined in the stratum $\mathcal{S}_{1}$ and represent two cross foliations of the same stratum at the same time.

We thus get three foliations, one on $\mathcal{S}_{0}$ and two on $\mathcal{S}_{1}$, that we can visualize in $\mathcal{S}_{0} \cap \mathcal{S}_{1}$ as represented in Fig. 15: 
- On $\mathcal{S}_{0}$ we define the $(\alpha, \xi)$-foliation and the leafs $\mathcal{Q}_{0, \alpha, \xi}$ in green (vertical lines) on Fig. 15. A path along one of these leaves called a free path.

- On $\mathcal{S}_{1}$ we define the $(\beta, \xi)$-foliation and the leafs $\mathcal{Q}_{1, \beta, \xi}$ in blue (thick dots) on Fig. 15. A path along one of these leaves called a manipulation path.

- On $\mathcal{S}_{1}$ we define the $(\alpha, \beta)$-foliation and the leafs $\mathcal{Q}_{2, \alpha, \beta}$ in red (thin dots) on Fig. 15. A path along one of these leaves called a locomotion path.

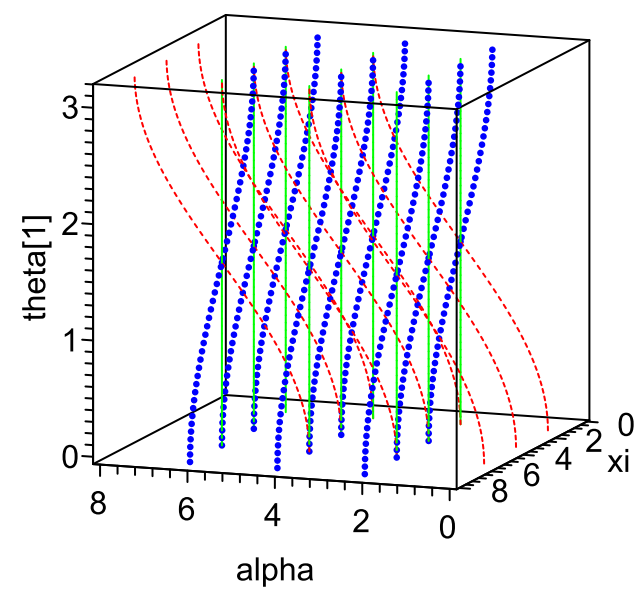

Fig. 15 The foliations of the three strata for the system $R_{3}$. In blue (thick dots) the $(\beta, \xi)-$ foliation, in red (thin dots) the $(\alpha, \beta)$-foliation, in green (vertical solid lines) the $(\alpha, \xi)$-foliation.

Proposition 3 If there exists for $R_{3}$ a collision-free path in unfoliated $\mathcal{S}_{1} \cap \operatorname{cl}\left(\mathcal{S}_{0}\right)$ from $q_{\text {initial }}$ to $q_{\text {final }}$ then there exists a collision-free finite sequence of free, manipulation, and locomotion paths that links $q_{\mathrm{initial}}$ and $q_{\mathrm{final}}$.

Proof Let us consider the 3D Cartesian space $\mathbb{R}^{2} \times(0, \pi)$ provided with the system of coordinates $\left(\alpha, \xi, \theta_{1}\right)$ in which we consider a compact subset $O$ and the families of functions

$$
\begin{aligned}
f_{\alpha, \beta}:(0, \pi) & \rightarrow \mathbb{R}^{3} \\
\theta_{1} & \mapsto\left\{\begin{array}{l}
\alpha \\
\beta-2 \cos \left(\theta_{1}\right) \quad \alpha, \beta \in \mathbb{R} \\
\theta_{1}
\end{array}\right.
\end{aligned}
$$

which represents the red (thin dots) foliation,

$$
\begin{aligned}
g_{\beta, \xi}:(0, \pi) & \rightarrow \mathbb{R}^{3} \\
\theta_{1} & \mapsto\left\{\begin{array}{l}
\beta+2 \cos \left(\theta_{1}\right) \\
\xi \\
\theta_{1}
\end{array} \quad \beta, \xi \in \mathbb{R}\right.
\end{aligned}
$$


which represents the blue (thick dots) foliation,

$$
\begin{aligned}
h_{\alpha, \xi}:(0, \pi) & \rightarrow \mathbb{R}^{3} \\
\theta_{1} & \mapsto\left\{\begin{array}{l}
\alpha \\
\xi \\
\theta_{1}
\end{array} \quad \alpha, \xi \in \mathbb{R}\right.
\end{aligned}
$$

which represents the green (vertical lines) foliation.

First, we prove that any collision-free path parallel to the $\alpha$ axis can be decomposed into a finite sequence of collision-free paths along the foliations. Let that $\alpha$-parallel path be defined by $\theta_{1}=\theta_{1_{0}}$ and $\xi=\xi_{0}$. The foliations $\left(g_{\beta, \xi_{0}}\right)_{\beta}$ and $\left(h_{\alpha, \xi_{0}}\right)_{\alpha}$ represent two foliations in the affine plan $\xi=\xi_{0}$, one strictly decreasing and one constant, for which we can directly apply Proposition 2. Thus, in that affine plan $\xi=\xi_{0}$, we can decompose the $\alpha$-parallel path into a finite sequence of blue and green paths.

Similarly we prove that any collision-free path parallel to the $\xi$ axis can be decomposed into a finite sequence of collision-free paths along the foliations. Let that $\xi$-parallel path be defined by $\theta_{1}=\theta_{1_{0}}$ and $\alpha=\alpha_{0}$. The foliations $\left(f_{\alpha_{0}, \beta}\right)_{\beta}$ and $\left(h_{\alpha_{0}, \xi}\right)_{\xi}$ represent two foliations in the affine plan $\alpha=\alpha_{0}$, one strictly increasing and one constant, for which we can directly apply Proposition 1. Thus in that affine plan $\alpha=\alpha_{0}$ we can decompose the $\xi$-parallel path into a finite sequence of red and green paths.

Any collision-free path parallel to the $\theta_{1}$ axis is already a green path in the foliation.

Now extending the same method that we used in the proof of Proposition 1, we can prove that any collision-free path in $\mathcal{S}_{0} \cap \mathcal{S}_{1}$ can be decomposed in a finite sequence of collision-free paths parallel to the axes $\alpha, \xi$ and $\theta_{1}$.

One important remark has to be made at this point. The motion that we get by this planning is a succession of isolated locomotion and manipulation motions, with either $\alpha=$ constant or $\xi=$ constant. However, we can plan a motion in which both $\alpha$ and $\xi$ are varying simultaneously, which would be equivalent to a locomotion-while-manipulating conceptual motion. This can be done simply by replacing one of the two foliations on $\mathcal{S}_{1}$ with a new foliation. Let us call it the $\left(\lambda_{1}, \lambda_{2}\right)$-foliation, $\lambda_{1}+\lambda_{2}=1$, for which we write a condition $\lambda_{1} \alpha+\lambda_{2} \xi=$ constant replacing one of the conditions $\alpha=$ constant or $\xi=$ constant. The $\left(\lambda_{1}, \lambda_{2}\right)$-foliation replacing one of the previous two on $\mathcal{S}_{1}$ makes it still possible to explore all the foliated space using the reduction property. Moreover, adding the $\left(\lambda_{1}, \lambda_{2}\right)$-foliation to the set of the previous three adds redundancy in the system and gives multiple solutions for the motion planning problem. Thus, it is also possible to synthesize a locomotion-while-manipulating motion.

Let us call a path through the $\left(\lambda_{1}, \lambda_{2}\right)$-foliation a locomotion-while-manipulation path. The previous remark translates into the following corollary:

Corollary 1 If there exists for $R_{3}$ a collision-free path in unfoliated $\mathcal{S}_{1} \cap \operatorname{cl}\left(\mathcal{S}_{0}\right)$ from $q_{\text {initial }}$ to $q_{\text {final }}$ then there exists

- a collision-free finite sequence of free, manipulation, and locomotion-whilemanipulation paths that links $q_{\text {initial }}$ and $q_{\text {final }}$.

- a collision-free finite sequence of free, locomotion, and locomotion-while-manipulation paths that links $q_{\text {initial }}$ and $q_{\text {final }}$. 
- a collision-free finite sequence of free, locomotion, manipulation, and locomotionwhile-manipulation paths that links $q_{\text {initial }}$ and $q_{\text {final }}$.

\section{Kinematic Control-Theoretic Approach}

In the previous section we have seen the underactuation of the robots as foliations in the C-space along which we need to cruise in order to reach our goal. In this section, we rather see this underactuation as a non-spanning distribution of control vector fields, our robots being considered as driftless stratified kinematic control systems. We strongly advise the reader to refer to the two main references [21] and [22] since all what follows builds on their result. The references $[32,14]$ can also prove useful for the reader unfamiliar with mathematical tools for nonholonomic motion planning (especially, the notions of Lie Brackets, distributions associated with control fields, Philip Hall basis of a Lie Algebra, formal exponential).

\subsection{Locomotion robot}

First let us consider the robot $R_{1}$.

The aim here is to generate a trajectory (time and space) (as opposed to path, i.e. only space, produced in the previous approach) using nonholonomic control techniques but without explicitly taking the obstacles into account. However, the philosophy remains the same: planning a sequence of transfer and transit trajectories in $\mathcal{S}_{1} \cap \operatorname{cl}\left(\mathcal{S}_{0}\right)$.

For this, we first need to model $R_{1}$ as a kinematic control system. Our kinematic control inputs are $u_{1}=\dot{\theta_{1}}$ and $u_{2}=\dot{\theta_{2}}$. No control input directly controls $\xi$.

The system is stratified in the sense defined in [21]. If we denote by $\Phi \in \mathscr{C}^{\infty}(\mathcal{C})$ the function that maps every configuration $q \in \mathcal{C}$ to the height of the end effector $h=\Phi(q)=y(q)$, then we can redefine $\mathcal{S}_{0}=\mathcal{C}$ as the top stratum and $\mathcal{S}_{1}=$ $\Phi^{-1}(\{0\})$ as the bottom stratum. We have the trivial inclusion chain $\mathcal{S}_{1} \subset \mathcal{S}_{0}$.

Two different equations of motion are acting on the two strata:

- On $\mathcal{S}_{0}$, the base is fixed and we can write

$$
\frac{d}{d t}\left(\begin{array}{c}
\xi \\
\theta_{1} \\
\theta_{2}
\end{array}\right)=\left(\begin{array}{l}
0 \\
1 \\
0
\end{array}\right) u_{1}+\left(\begin{array}{l}
0 \\
0 \\
1
\end{array}\right) u_{2}
$$

- On $\mathcal{S}_{1}$, the end effector is fixed as we consider a non-sliding contact, and thus the equation of motion is written

$$
\frac{d}{d t}\left(\begin{array}{c}
\xi \\
\theta_{1} \\
\theta_{2}
\end{array}\right)=\left(\begin{array}{c}
2 l \sin \left(\theta_{1}\right) \\
1 \\
-2
\end{array}\right) u_{1}
$$

We can rewrite those two equations using the formalism of driftless control theory [36]. Let $x=\left(\xi, \theta_{1}, \theta_{2}\right)^{T}$ denote the state of our kinematic system (Note: for the remaining of this section $x$ denotes the state of the system as usual in control theory and not the $x$-coordinate of the end-effector). Let $g_{0,1}(x)=\frac{\partial}{\partial \theta_{1}}, g_{0,2}(x)=$ 
$\frac{\partial}{\partial \theta_{2}}$ be the two control fields acting on $\mathcal{S}_{0}$ and $g_{1,1}(x)=2 l \sin \left(\theta_{1}\right) \frac{\partial}{\partial \xi}+\frac{\partial}{\partial \theta_{1}}-2 \frac{\partial}{\partial \theta_{2}}$. Then our stratified driftless system is modelled by the two equations:

$$
\begin{array}{ll}
\dot{x}=g_{0,1}(x) u_{1}+g_{0,2}(x) u_{2}, & , x \in \mathcal{S}_{0} \\
\dot{x}=g_{1,1}(x) u_{1} & , x \in \mathcal{S}_{1}
\end{array}
$$

Let us study the controllability of our system.

Proposition 4 The underactuated kinematic control system $R_{1}$ is small time locally controllable in $\operatorname{int}\left(\mathcal{C}_{\text {free }}\right)$

Proof We consider $x_{0} \in \mathcal{S}_{1}$ an element from the bottom stratum. Let

$$
\begin{aligned}
& \left.\Delta_{\mathcal{S}_{0}}\right|_{x_{0}}=\operatorname{span}\left\{g_{0,1}\left(x_{0}\right), g_{0,2}\left(x_{0}\right)\right\} \\
& \left.\Delta_{\mathcal{S}_{1}}\right|_{x_{0}}=\operatorname{span}\left\{g_{1,1}\left(x_{0}\right)\right\}
\end{aligned}
$$

be the distributions associated with the control fields of each stratum and $\left.\bar{\Delta}_{\mathcal{S}_{0}}\right|_{x_{0}}$ and $\left.\bar{\Delta}_{\mathcal{S}_{1}}\right|_{x_{0}}$ be their involutive closure under Lie Bracketting. Since $\left[g_{0,1}, g_{0,2}\right]=0$ we have

$$
\begin{aligned}
& \left.\bar{\Delta}_{\mathcal{S}_{0}}\right|_{x_{0}}=\operatorname{span}\left\{g_{0,1}\left(x_{0}\right), g_{0,2}\left(x_{0}\right)\right\} \\
& \bar{\Delta}_{\mathcal{S}_{1} \mid x_{0}}=\operatorname{span}\left\{g_{1,1}\left(x_{0}\right)\right\}
\end{aligned}
$$

Therefore, for each $x_{0} \in \mathcal{S}_{1}$ such that $\theta_{1} \neq k \pi$

$$
\left.\bar{\Delta}_{\mathcal{S}_{0}}\right|_{x_{0}}+\left.\bar{\Delta}_{\mathcal{S}_{1}}\right|_{x_{0}}=\operatorname{span}\left\{g_{0,1}\left(x_{0}\right), g_{0,2}\left(x_{0}\right), g_{1,1}\left(x_{0}\right)\right\}=T_{x_{0}} \mathcal{C}
$$

and thus following the controllability theorem of [21] the system is small time locally controllable from $x_{0}$.

Now let us address the issue of gait controllability. We consider the cyclic gait

$$
\mathcal{G}=\left(\mathcal{S}_{1}, \mathcal{S}_{0}, \mathcal{S}_{1}\right)
$$

in which the robot alternatively lifts its end-effector off the ground and then put it back on the ground.

Proposition $5 R_{1}$ is gait-controllable with the gait $\mathcal{G}$.

Proof We construct the gait distribution as follows:

$$
\begin{aligned}
& \mathcal{D}_{1}=\left.\bar{\Delta}_{\mathcal{S}_{1}}\right|_{x_{0}} \\
& \mathcal{D}_{2}=\mathcal{D}_{1}+\left.\bar{\Delta}_{\mathcal{S}_{0}}\right|_{x_{0}}=T_{x_{0}} \mathcal{C} \\
& \mathcal{D}_{3}=\left(\mathcal{D}_{2} \cap T_{x_{0}} \mathcal{S}_{1}\right)+\left.\bar{\Delta}_{\mathcal{S}_{1}}\right|_{x_{0}}
\end{aligned}
$$

We can parametrize $\mathcal{S}_{1}$ by the equations

$$
\mathcal{S}_{1}:\left\{\begin{array}{l}
\xi=\xi \\
\theta_{1}=\theta_{1} \\
\theta_{2}=-2 \theta_{1}
\end{array}\right.
$$

which allows us to write

$$
T_{x_{0}} \mathcal{S}_{1}=\operatorname{span}\left\{\frac{\partial}{\partial \xi}, \frac{\partial}{\partial \theta_{1}}-2 \frac{\partial}{\partial \theta_{2}}\right\}
$$

We can see that $g_{1,1}\left(x_{0}\right) \in T_{x_{0}} \mathcal{S}_{1}$ and thus $\mathcal{D}_{3}=T_{x_{0}} \mathcal{S}_{1}$ meaning that $\operatorname{dim}\left(\mathcal{D}_{3}\right)=$ $\operatorname{dim}\left(T_{x_{0}} \mathcal{S}_{1}\right)$, which proves, following [21]'s result, the gait controllability of $\mathcal{G}$. 
We want now to plan a motion from an initial state $q_{\text {initial }}=\left(\xi_{i}, \theta_{1_{i}},-2 \theta_{1_{i}}\right)^{T} \in$ $\mathcal{S}_{1}$ to a goal state $q_{\text {final }}=\left(\xi_{f}, \theta_{1_{f}},-2 \theta_{1_{f}}\right)^{T} \in \mathcal{S}_{1}$. To do so, we first construct a stratified extended system on $\mathcal{S}_{1}$ by constructing a vector field from $\Delta_{\mathcal{S}_{0}}$ that is tangent to $\mathcal{S}_{1}$. The vector field we consider here is $g_{1,2}=g_{0,1}-2 g_{0,2}=\frac{\partial}{\partial \theta_{1}}-2 \frac{\partial}{\partial \theta_{2}}$, so that our system becomes, on the bottom stratum $\mathcal{S}_{1}$ :

$$
\dot{x}=g_{1,1}(x) u_{1}+g_{1,2}(x) u_{2}
$$

We then extend the system by adding a vector field from the Lie Algebra of the two control fields we now have on $\mathcal{S}_{1}$ to better condition the system. We get the following stratified system on $\mathcal{S}_{1}$ :

$$
\dot{x}=b_{1} v_{1}+b_{2} v_{2}+b_{3} v_{3}
$$

where

$$
\begin{aligned}
& b_{1}=g_{1,1} \\
& b_{2}=g_{1,2} \in \Delta_{\mathcal{S}_{0}} \cap T \mathcal{S}_{1} \\
& b_{3}=\left[b_{1}, b_{2}\right]=2 l \cos \left(\theta_{1}\right) \frac{\partial}{\partial \xi}
\end{aligned}
$$

We then solve this system for the fictitious inputs $v_{1}, v_{2}, v_{3}$ given a straight line trajectory linking $q_{\text {initial }}$ and $q_{\text {final }}$ :

$$
\gamma(t)=\left(\gamma_{\xi}(t), \gamma_{\theta_{1}}(t),-2 \gamma_{\theta_{1}}(t)\right)^{T}
$$

where

$$
\begin{aligned}
& \gamma_{\xi}(t)=\xi_{i}+\Delta \xi . t \\
& \gamma_{\theta_{1}}(t)=\theta_{1_{i}}+\Delta \theta_{1} . t \\
& \Delta \xi=\xi_{f}-\xi_{i} \\
& \Delta \theta_{1}=\theta_{1_{f}}-\theta_{1_{i}}
\end{aligned}
$$

meaning that we solve

$$
\dot{\gamma}(t)=b_{1}(\gamma(t)) v_{1}+b_{2}(\gamma(t)) v_{2}+b_{3}(\gamma(t)) v_{3}
$$

which requires pseudo inverting a matrix

$$
\left(\begin{array}{c}
\Delta \xi \\
\Delta \theta_{1} \\
-2 \Delta \theta_{1}
\end{array}\right)=\left(\begin{array}{ccc}
2 l \sin \left(\gamma_{\theta_{1}}(t)\right) & 0 & 2 l \cos \left(\gamma_{\theta_{1}}(t)\right) \\
1 & 1 & 0 \\
-2 & -2 & 0
\end{array}\right)\left(\begin{array}{c}
v_{1} \\
v_{2} \\
v_{3}
\end{array}\right)
$$

One solution for this system is

$$
\left(\begin{array}{l}
v_{1}(t) \\
v_{2}(t) \\
v_{3}(t)
\end{array}\right)=\left(\begin{array}{c}
0 \\
\Delta \theta_{1} \\
\frac{\Delta \xi}{2 l \cos \left(\gamma_{\theta_{1}}(t)\right)}
\end{array}\right)
$$

given these inputs we solve the formal ordinary differential equation in a backward Philip Hall ${ }^{2}$ basis of the Lie Algebra generated by $b_{1}, b_{2}, b_{3}$ (which happens to be $\left.\left(b_{1}, b_{2}, b_{3}\right)\right)$

$$
\dot{S}(t)=S(t)\left(b_{1} v_{1}+b_{2} v_{2}+b_{3} v_{3}\right)
$$

\footnotetext{
2 http://planning.cs.uiuc.edu/node834.html
} 
for which we search for a solution of the form

$$
S(t)=e^{h_{3}(t) b_{3}} e^{h_{2}(t) b_{2}} e^{h_{1}(t) b_{1}}
$$

by expanding the formal exponentials to second order (e.g. $e^{h_{1}(t) b_{1}}=I+h_{1}(t) b_{1}+$ $\frac{h_{1}^{2}}{2}(t) b_{1}^{2}+\cdots$, where the terms of the form $b_{i}^{k}$ are partial derivative operators (and not vector fields), and by equating the resulting coefficients of the $b_{i}$ 's in (45), we get the set of equations for the $h_{i}$ functions:

$$
\left\{\begin{array}{l}
\dot{h}_{1}(t)=v_{1} \\
\dot{h}_{2}(t)=v_{2} \\
\dot{h}_{3}(t)=h_{1}(t) v_{2}+v_{3}
\end{array}\right.
$$

with the initial conditions $h_{i}(0)=0$ for $i=1,2,3$.

Integrating those equations gives us the "durations" for following each flow of the control field:

$$
\begin{aligned}
& h_{1}(1)=0 \\
& h_{2}(1)=\Delta \theta_{1} \\
& h_{3}(1)=\frac{\Delta \xi}{2 l \Delta \theta_{1}} \ln \left|\frac{1}{\cos \left(\theta_{1_{f}}\right)}+\tan \left(\theta_{1_{f}}\right)\right|
\end{aligned}
$$

if $\Delta \theta_{1} \neq 0$, or

$$
\begin{aligned}
& h_{1}(1)=0 \\
& h_{2}(1)=0 \\
& h_{3}(1)=\frac{\Delta \xi}{2 l}
\end{aligned}
$$

if $\Delta \theta_{1}=0$.

Let's consider the case $\Delta \theta_{1} \neq 0$.

If we denote $\phi_{t}^{b_{i}}$ as the flow associated with the field $b_{i}$, the solution should thus be: follow $\phi_{t}^{b_{1}}$ for $t=0 s$, then follow $\phi_{t}^{b_{2}}$ for $t=\Delta \theta_{1} s$, then follow $\phi_{t}^{b_{3}}$ for $t=\frac{\Delta \xi}{2 l \Delta \theta_{1}} \ln \left|\frac{1}{\cos \left(\theta_{1_{f}}\right)}+\tan \left(\theta_{1_{f}}\right)\right| s$. However, the flow associated with $b_{3}=\left[b_{1}, b_{2}\right]$ starting from $x_{0}$ could be rewritten, for $t>0$ :

$$
\phi_{t}^{\left[b_{1}, b_{2}\right]}\left(x_{0}\right)=\phi_{\sqrt{t}}^{-b_{2}} \circ \phi_{\sqrt{t}}^{-b_{1}} \circ \phi_{\sqrt{t}}^{b_{2}} \circ \phi_{\sqrt{t}}^{b_{1}}\left(x_{0}\right)+\mathcal{O}(t)
$$

Finally, let us denote $\alpha u_{i}$ the command consisting in letting $u_{i}=1$ for $\alpha$ seconds if $\alpha \geq 0$ and $u_{i}=-1$ for $-\alpha$ seconds if $\alpha<0$, and denote two successive controls by the overloaded concatenation operator $\circ$ as in [22]. We denote control laws as functions $s$ mapping time to the controls $s: t \mapsto\left(u_{1}(t), u_{2}(t)\right)$. More formally, the notation $s=\alpha_{1} u_{1} \circ \alpha_{2} u_{2}$ will denote the control law

$$
s: t \mapsto \begin{cases}\left(u_{1}(t), u_{2}(t)\right)=\left(\operatorname{sgn}\left(\alpha_{1}\right), 0\right), & 0 \leq t<\left|\alpha_{1}\right| \\ \left(u_{1}(t), u_{2}(t)\right)=\left(0, \operatorname{sgn}\left(\alpha_{2}\right)\right), & \left|\alpha_{1}\right| \leq t<\left|\alpha_{1}\right|+\left|\alpha_{2}\right|\end{cases}
$$

where sgn denotes the sign function.

Hence, using these notations for our motion planning problem, we get our final sequence of commands (supposing for example that $\Delta \xi \geq 0$ ):

$$
s=0 u_{1} \circ \Delta \theta_{1} u_{2} \circ \sqrt{\frac{\Delta \xi}{2 l \Delta \theta_{1}} \ln \left|\frac{1}{\cos \left(\theta_{1_{f}}\right)}+\tan \left(\theta_{1_{f}}\right)\right|}\left(u_{1} \circ u_{2} \circ-u_{1} \circ-u_{2}\right)
$$


applied to the flows

$$
\phi_{t}^{b_{1}}\left(x_{0}\right)=\left(\begin{array}{c}
2\left(\frac{\xi_{0}}{2}+\cos \left(\theta_{1_{0}}\right)-\cos \left(t+\theta_{1_{0}}\right)\right) \\
t+\theta_{1_{0}} \\
-2\left(t+\theta_{1_{0}}\right)
\end{array}\right)
$$

and

$$
\phi_{t}^{b_{2}}\left(x_{0}\right)=\left(\begin{array}{c}
\xi_{0} \\
t+\theta_{1_{0}} \\
-2\left(t+\theta_{1_{0}}\right)
\end{array}\right)
$$

In the case $\Delta \theta_{1}=0$ the solution is simply

$$
s=\sqrt{\frac{\Delta \xi}{2 l}}\left(u_{1} \circ u_{2} \circ-u_{1} \circ-u_{2}\right)
$$

The solution is pictured in Fig. 16 in which the red curve represents the final output for an initial trajectory that is the black vertical line from 0 to 10 .

Note that we do not reach the goal exactly, but with a bounded error [32,22]. The bound on the error allows us to reiterate this algorithm from the reached state as a new initial state until we reach the goal with a desired precision.

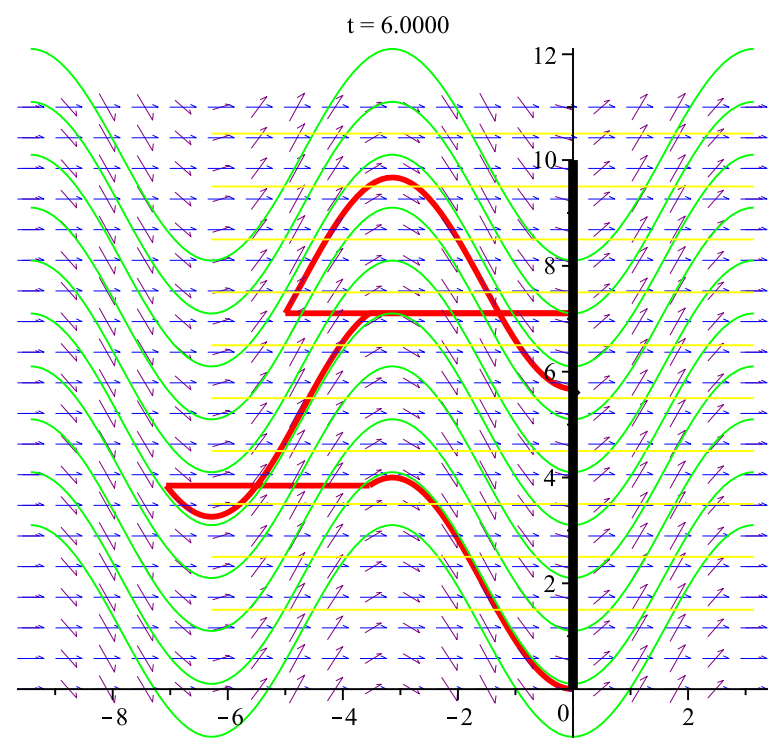

Fig. 16 Solution in the $\left(\xi, \theta_{1}\right)$ plan. The horizontal axis is the $\xi$ axis and the vertical axis is the The initial trajectory is the $\theta_{1}$ axis. the thick black vertical segment drawn on $\xi$ axis. The resulting solution is the red trajectory. In blue the $g_{1,2}$ control field, with its integral curves in yellow. In purple the $g_{1,1}$ control field, with its integral curves in green. 
4.2 Manipulation robot

For $R_{2}$ we have similar properties to $R_{1}$. We just need to replace the variable $\xi$ by the variable $\alpha$. So let us consider the coordinate chart $\left(\alpha, \theta_{1}, \theta_{2}\right)$ in our configuration space manifold.

The equations of motion that are acting on the two strata are as follows:

- On $\mathcal{S}_{0}$, the platform is fixed and we can write

$$
\frac{d}{d t}\left(\begin{array}{c}
\alpha \\
\theta_{1} \\
\theta_{2}
\end{array}\right)=\left(\begin{array}{l}
0 \\
1 \\
0
\end{array}\right) u_{1}+\left(\begin{array}{l}
0 \\
0 \\
1
\end{array}\right) u_{2}
$$

- On $\mathcal{S}_{1}$, the end-effector is fixed in the platform's inertial frame as we consider a non-sliding contact, and thus the equation of motion is written

$$
\frac{d}{d t}\left(\begin{array}{c}
\alpha \\
\theta_{1} \\
\theta_{2}
\end{array}\right)=\left(\begin{array}{c}
-2 l \sin \left(\theta_{1}\right) \\
1 \\
-2
\end{array}\right) u_{1}
$$

The stratified driftless system is modelled by the two equations:

$$
\begin{array}{ll}
\dot{x}=g_{0,1}(x) u_{1}+g_{0,2}(x) u_{2}, & x \in \mathcal{S}_{0} \\
\dot{x}=g_{1,1}(x) u_{1} & , x \in \mathcal{S}_{1}
\end{array}
$$

where

$$
\begin{aligned}
& g_{0,1}(x)=\frac{\partial}{\partial \theta_{1}} \\
& g_{0,2}(x)=\frac{\partial}{\partial \theta_{2}} \\
& g_{1,1}(x)=-2 l \sin \left(\theta_{1}\right) \frac{\partial}{\partial \xi}+\frac{\partial}{\partial \theta_{1}}-2 \frac{\partial}{\partial \theta_{2}}
\end{aligned}
$$

Proposition 6 The underactuated kinematic control system $R_{2}$ is small time locally controllable in $\operatorname{int}\left(\mathcal{C}_{\text {free }}\right)$

Proof The proof follows the same pattern as the proof of Proposition 4.

Let's consider the gait $\mathcal{G}=\left(\mathcal{S}_{1}, \mathcal{S}_{0}, \mathcal{S}_{1}\right)$

Proposition $7 \quad R_{2}$ is gait-controllable with the gait $\mathcal{G}$.

Proof The proof follows the same pattern as the proof of Proposition 5.

We want now to plan a motion from a given $q_{\text {initial }}=\left(\alpha_{i}, \theta_{1_{i}},-2 \theta_{1_{i}}\right)^{T}$ to a given $q_{\text {final }}=\left(\alpha_{f}, \theta_{1_{f}},-2 \theta_{1_{f}}\right)$ in $\mathcal{C}_{\text {free }}$.

Using the exact same method as for $R_{1}$, for $\Delta \theta_{1} \neq 0$ and supposing for example that $\Delta \alpha \geq 0$, we get the solution:

$$
s=0 u_{1} \circ \Delta \theta_{1} u_{2} \circ \sqrt{\frac{\Delta \alpha}{2 l \Delta \theta_{1}} \ln \left|\frac{1}{\cos \left(\theta_{1_{f}}\right)}+\tan \left(\theta_{1_{f}}\right)\right|}\left(u_{2} \circ u_{1} \circ-u_{2} \circ-u_{1}\right)
$$

applied to the flows

$$
\phi_{t}^{b_{1}}\left(x_{0}\right)=\left(\begin{array}{c}
2\left(\frac{\alpha_{0}}{2}+\cos \left(t+\theta_{1_{0}}\right)-\cos \left(\theta_{1_{0}}\right)\right) \\
t+\theta_{1_{0}} \\
-2\left(t+\theta_{1_{0}}\right)
\end{array}\right)
$$


and

$$
\phi_{t}^{b_{2}}\left(x_{0}\right)=\left(\begin{array}{c}
\xi_{0} \\
t+\theta_{1_{0}} \\
-2\left(t+\theta_{1_{0}}\right)
\end{array}\right)
$$

For $\Delta \theta_{1}=0$ we get:

$$
s=\sqrt{\frac{\Delta \alpha}{2 l}}\left(u_{2} \circ u_{1} \circ-u_{2} \circ-u_{1}\right)
$$

\section{$4.3 \mathrm{~L} \& \mathrm{M}$ robot}

The robot $R_{3}$, with the switching modes control strategy introduced in Section 3, can also be modelled as a stratified system.

Let us first see why $R_{3}$ cannot be directly modelled as a driftless stratified system if we do not consider this switching strategy. In this case, when the rubber end-effector is in contact at a fixed location in the platform's frame $\beta=$ constant, then the system evolves in the submanifold defined by the implicit equation:

$$
\xi+2 l \cos \left(\theta_{1}\right)+\beta=\alpha
$$

Taking the derivative with respect to time $t$ leads:

$$
\dot{\xi}-2 l \sin \left(\theta_{1}\right) \dot{\theta_{1}}=\dot{\alpha}
$$

i.e.

$$
\left(\begin{array}{cccc}
1 & 0 & 0 & -1 \\
0 & 1 & 0 & 0 \\
0 & 0 & 1 & 0
\end{array}\right)\left(\begin{array}{c}
\dot{\xi} \\
\dot{\theta}_{1} \\
\dot{\theta}_{2} \\
\dot{\alpha}
\end{array}\right)=\left(\begin{array}{c}
2 l \sin \left(\theta_{1}\right) \\
1 \\
-2
\end{array}\right) \theta_{1}
$$

Writing $\dot{\theta_{1}}=u_{1}$ we get a system of the form

$$
A \dot{x}=\sum_{i} g_{i}(x) u_{i}
$$

where $A=\left(\begin{array}{cccc}1 & 0 & 0 & -1 \\ 0 & 1 & 0 & 0 \\ 0 & 0 & 1 & 0\end{array}\right)$ is a non invertible (non square) matrix and thus the system cannot be written in the desired form

$$
\dot{x}=\sum_{i} g_{i}(x) u_{i}
$$

Now back to the switching control strategy. The equations of motions acting on the two strata are:

- on $\mathcal{S}_{0}$ :

$$
\frac{d}{d t}\left(\begin{array}{c}
\xi \\
\theta_{1} \\
\theta_{2} \\
\alpha
\end{array}\right)=\left(\begin{array}{l}
0 \\
1 \\
0 \\
0
\end{array}\right) u_{1}+\left(\begin{array}{l}
0 \\
0 \\
1 \\
0
\end{array}\right) u_{2}
$$


- on $\mathcal{S}_{1}$, in manipulation state:

$$
\frac{d}{d t}\left(\begin{array}{c}
\xi \\
\theta_{1} \\
\theta_{2} \\
\alpha
\end{array}\right)=\left(\begin{array}{c}
0 \\
1 \\
-2 \\
-2 l \sin \left(\theta_{1}\right)
\end{array}\right) u_{1}
$$

- on $\mathcal{S}_{1}$, in locomotion state:

$$
\frac{d}{d t}\left(\begin{array}{c}
\xi \\
\theta_{1} \\
\theta_{2} \\
\alpha
\end{array}\right)=\left(\begin{array}{c}
2 l \sin \left(\theta_{1}\right) \\
1 \\
-2 \\
0
\end{array}\right) u_{1}
$$

As we can see, two different equations of motion are acting on the bottom stratum $\mathcal{S}_{1}$. They correspond to two control vector fields defined on $\mathcal{S}_{1}$. Since the solution produced by the method of [22] consists in following the vector fields sequentially and never a linear combination of the vector fields, we can use it for $R_{3}$ to produce the control sequence with the state-switching control nested in the solution.

We want to steer the system from a given $q_{\text {initial }}=\left(\xi_{i}, \theta_{1_{i}},-2 \theta_{1_{i}}, \alpha_{i}\right)^{T}$ to a given $q_{\text {final }}=\left(\xi_{f}, \theta_{1_{f}},-2 \theta_{1_{f}}, \alpha_{f}\right)^{T}$. We first derive equation the stratified driftless system on the bottom stratum:

$$
\dot{x}=g_{1}(x) u_{1}+g_{2}(x) u_{2}+g_{3}(x) u_{3}
$$

with

$$
\begin{aligned}
& g_{1}(x)=\left(\begin{array}{c}
0 \\
1 \\
-2 \\
0
\end{array}\right), g_{2}(x)=\left(\begin{array}{c}
2 l \sin \left(\theta_{1}\right) \\
1 \\
-2 \\
0
\end{array}\right) \\
& g_{3}(x)=\left(\begin{array}{c}
0 \\
1 \\
-2 \\
-2 l \sin \left(\theta_{1}\right)
\end{array}\right)
\end{aligned}
$$

We then extend the system by adding vector fields from $\operatorname{Lie}\left(g_{1}, g_{2}, g_{3}\right)$ :

$$
\dot{x}=b_{1} v_{1}+b_{2} v_{2}+b_{3} v_{3}+b_{4} v_{4}+b_{5} v 5
$$

where

$$
\begin{aligned}
& b_{1}=g_{1} \\
& b_{2}=g_{2} \\
& b_{3}=g_{3} \\
& b_{4}=\left[g_{1}, g_{2}\right]=\left(\begin{array}{c}
2 l \cos \left(\theta_{1}\right) \\
0 \\
0 \\
0
\end{array}\right) \\
& b_{5}=\left[g_{1}, g_{3}\right]=\left(\begin{array}{c}
0 \\
0 \\
0 \\
-2 l \cos \left(\theta_{1}\right)
\end{array}\right)
\end{aligned}
$$


Note: we stop at second order and we do not need to add $\left[g_{2}, g_{3}\right]=b_{5}-b_{4}$. We then we solve this system for the fictitious inputs $v_{1}, v_{2}, v_{3}, v_{4}, v_{5}$ given a straight line trajectory linking $q_{\text {initial }}$ and $q_{\text {final }}$ :

$$
\gamma(t)=\left(\gamma_{\xi}(t), \gamma_{\theta_{1}}(t),-2 \gamma_{\theta_{1}}(t), \gamma_{\alpha}(t)\right)^{T}
$$

where

$$
\begin{aligned}
& \gamma_{\xi}(t)=\xi_{i}+\Delta \xi . t \\
& \gamma_{\alpha}(t)=\alpha_{i}+\Delta \alpha . t \\
& \gamma_{\theta_{1}}(t)=\theta_{1_{i}}+\Delta \theta_{1} . t \\
& \Delta \xi=\xi_{f}-\xi_{i} \\
& \Delta \alpha=\alpha_{f}-\alpha_{i} \\
& \Delta \theta_{1}=\theta_{1_{f}}-\theta_{1_{i}}
\end{aligned}
$$

We solve

$$
\dot{\gamma}(t)=b_{1}(\gamma(t)) v_{1}+b_{2}(\gamma(t)) v_{2}+b_{3}(\gamma(t)) v_{3}+b_{4}(\gamma(t)) v_{4}+b_{5}(\gamma(t)) v_{5}
$$

which requires pseudo inverting the matrix

$$
\left(\begin{array}{c}
\Delta \xi \\
\Delta \theta_{1} \\
-2 \Delta \theta_{1} \\
\Delta \alpha
\end{array}\right)=\left(\begin{array}{ccccc}
0 & 2 l \sin \left(\gamma_{\theta_{1}}\right) & 0 & 2 l \cos \left(\gamma_{\theta_{1}}\right) & 0 \\
1 & 1 & 1 & 0 & 0 \\
-2 & -2 & -2 & 0 & 0 \\
0 & 0 & -2 l \sin \left(\gamma_{\theta_{1}}\right) & 0 & -2 l \cos \left(\gamma_{\theta_{1}}\right)
\end{array}\right)\left(\begin{array}{l}
v_{1} \\
v_{2} \\
v_{3} \\
v_{4} \\
v_{5}
\end{array}\right)
$$

One solution for this system

$$
\left(\begin{array}{l}
v_{1}(t) \\
v_{2}(t) \\
v_{3}(t) \\
v_{4}(t) \\
v_{5}(t)
\end{array}\right)=\left(\begin{array}{c}
\Delta \theta_{1} \\
0 \\
0 \\
\frac{\Delta \xi}{2 l \cos \left(\gamma_{\theta_{1}}(t)\right)} \\
-\frac{\Delta \alpha}{2 l \cos \left(\gamma_{\theta_{1}}(t)\right)}
\end{array}\right)
$$

given these inputs we solve the formal ordinary differential equation in a backward Philip Hall basis of the Lie Algebra generated by $b_{1}, b_{2}, b_{3}, b_{4}, b_{5}$ which is also $\left(b_{1}, b_{2}, b_{3}, b_{4}, b_{5}\right)$

$$
\dot{S}(t)=S(t)\left(b_{1} v_{1}+b_{2} v_{2}+b_{3} v_{3}+b_{4} v_{4}+b_{5} v_{5}\right)
$$

for which we search for a solution of the form

$$
S(t)=e^{h_{5}(t) b_{5}} e^{h_{4}(t) b_{4}} e^{h_{3}(t) b_{3}} e^{h_{2}(t) b_{2}} e^{h_{1}(t) b_{1}}
$$

by developing the formal exponentials to second order, we get the set of equations for the $h_{i}$ functions:

$$
\left\{\begin{array}{l}
\dot{h}_{1}=v_{1} \\
\dot{h}_{2}=v_{2} \\
\dot{h}_{3}=v_{3} \\
-\dot{h}_{2} h_{1}+\dot{h}_{3} h_{2}+\dot{h}_{4}=v_{4} \\
-\dot{h}_{3} h_{1}-\dot{h}_{3} h_{2}+\dot{h}_{5}=v_{5}
\end{array}\right.
$$

with the initial conditions $h_{i}(0)=0$ for $i=1,2,3,4,5$. 
Integrating those equations gives us the "durations" for following each flow of the control field:

$$
\begin{aligned}
& h_{1}(1)=\Delta \theta_{1} \\
& h_{2}(1)=0 \\
& h_{3}(1)=0 \\
& h_{4}(1)=\frac{\Delta \xi}{2 l \Delta \theta_{1}} \ln \left|\frac{1}{\cos \left(\theta_{1_{f}}\right)}+\tan \left(\theta_{1_{f}}\right)\right| \\
& h_{5}(1)=-\frac{\Delta \alpha}{2 l \Delta \theta_{1}} \ln \left|\frac{1}{\cos \left(\theta_{1_{f}}\right)}+\tan \left(\theta_{1_{f}}\right)\right|
\end{aligned}
$$

if $\Delta \theta_{1} \neq 0$, or

$$
\begin{aligned}
& h_{1}(1)=0 \\
& h_{2}(1)=0 \\
& h_{3}(1)=0 \\
& h_{4}(1)=\frac{\Delta \xi}{2 l} \\
& h_{5}(1)=\frac{\Delta \alpha}{2 l}
\end{aligned}
$$

if $\Delta \theta_{1}=0$.

Finally, for $\Delta \theta_{1} \neq 0$ and supposing for example that $\Delta \xi \geq 0$ and $\Delta \alpha \geq 0$, we get the solution:

$$
\begin{aligned}
s=\Delta \theta_{1} u_{1} & \circ 0 u_{2} \circ 0 u_{3} \\
& \circ \sqrt{\frac{\Delta \xi}{2 l \Delta \theta_{1}} \ln \left|\frac{1}{\cos \left(\theta_{1_{f}}\right)}+\tan \left(\theta_{1_{f}}\right)\right|}\left(u_{1} \circ u_{2} \circ-u_{1} \circ-u_{2}\right) \\
& \circ \sqrt{\frac{\Delta \alpha}{2 l \Delta \theta_{1}} \ln \left|\frac{1}{\cos \left(\theta_{1_{f}}\right)}+\tan \left(\theta_{1_{f}}\right)\right|}\left(u_{3} \circ u_{1} \circ-u_{3} \circ-u_{1}\right)
\end{aligned}
$$

applied to the flows

$$
\begin{aligned}
& \phi_{t}^{b_{1}}\left(x_{0}\right)=\left(\begin{array}{c}
\xi_{0} \\
t+\theta_{1_{0}} \\
-2\left(t+\theta_{1_{0}}\right) \\
\alpha_{0}
\end{array}\right) \\
& \phi_{t}^{b_{2}}\left(x_{0}\right)=\left(\begin{array}{c}
2\left(\frac{\xi_{0}}{2}-\cos \left(t+\theta_{1_{0}}\right)+\cos \left(\theta_{1_{0}}\right)\right) \\
t+\theta_{1_{0}} \\
-2\left(t+\theta_{1_{0}}\right) \\
\alpha_{0}
\end{array}\right) \\
& \phi_{t}^{b_{3}}\left(x_{0}\right)=\left(\begin{array}{c}
\xi_{0} \\
t+\theta_{1_{0}} \\
-2\left(t+\theta_{1_{0}}\right) \\
2\left(\frac{\alpha_{0}}{2}+\cos \left(t+\theta_{1_{0}}\right)-\cos \left(\theta_{1_{0}}\right)\right)
\end{array}\right)
\end{aligned}
$$

For $\Delta \theta_{1}=0$ we get:

$$
s=\sqrt{\frac{\Delta \xi}{2 l}}\left(u_{1} \circ u_{2} \circ-u_{1} \circ-u_{2}\right) \circ \sqrt{\frac{\Delta \alpha}{2 l}}\left(u_{3} \circ u_{1} \circ-u_{3} \circ-u_{1}\right)
$$

The solution is pictured in Figs. 17 and 18. 


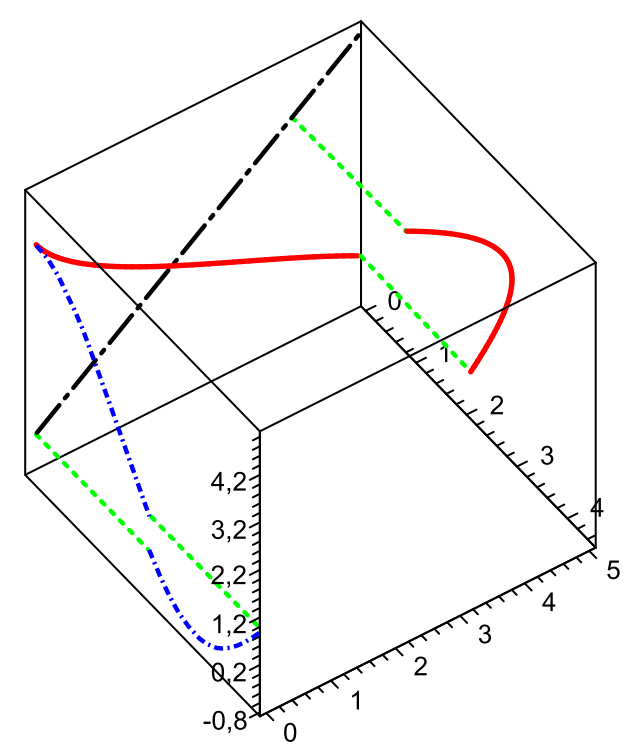

Fig. 17 Trajectory planning for $R_{3}$ in the $\left(\xi, \theta_{1}, \alpha\right)$ space. The bottom left horizontal axis is the $\xi$ axis, the bottom right horizontal axis is the $\theta_{1}$ axis, the vertical axis is the $\alpha$ axis. The initial trajectory, which violates the foliation, is the black (point/big-dashed) diagonal segment on the left-back face of the cube, the resulting trajectory is the red(line)-blue(pointdashed)-green(dashed) trajectory that follows the foliations. The startpoint of the motion is the intersection of the green segment and black diagonal segment in the bottom left, the endpoint is the intersection of the green segment and black black segment in the top right. The green segments are motions along the $(\alpha, \xi)$-foliation, the blue segments along the $(\beta, \xi)$ foliation, and finally the red segments along the $(\alpha, \beta)$-foliation (the colors used for the three foliations are the same as in Fig. 15).

\section{Dynamic Trajectory Planning Approach}

In the previous sections, we were primarily concerned by geometric path planning, even though Section 4 tackled the problem from a kinematic trajectory planning perspective. In this section, the objective is to generate torque-driven dynamically valid trajectories in the state space $T \mathcal{C}$ (the tangent bundle of the smooth manifold C).

\subsection{Locomotion robot}

First let us study the case of the robot $R_{1}$. We would like to generate dynamically valid trajectories (open-loop control laws) for both the transfer and the transit paths.

Problem 2 Given $\left(q_{\text {initial }}, \dot{q}_{\text {initial }}\right),\left(q_{\text {final }}, \dot{q}_{\text {final }}\right) \in T \mathcal{C}_{\text {free }}$ and a geometric path $p:[0,1] \rightarrow \mathcal{C}_{\text {free }}$ such that $p(0)=q_{\text {initial }}$ and $p(1)=q_{\text {final }}$, find $t_{f} \in \mathbb{R}$ and a re-parametrization of $\operatorname{Tr}(p) \gamma:\left[0, t_{f}\right] \rightarrow \mathcal{C}_{\text {free }}$ such that $\gamma$ realizes the dynamics equations of motion of $R_{1}$ along the path, under a Coulomb friction model hypothesis. 

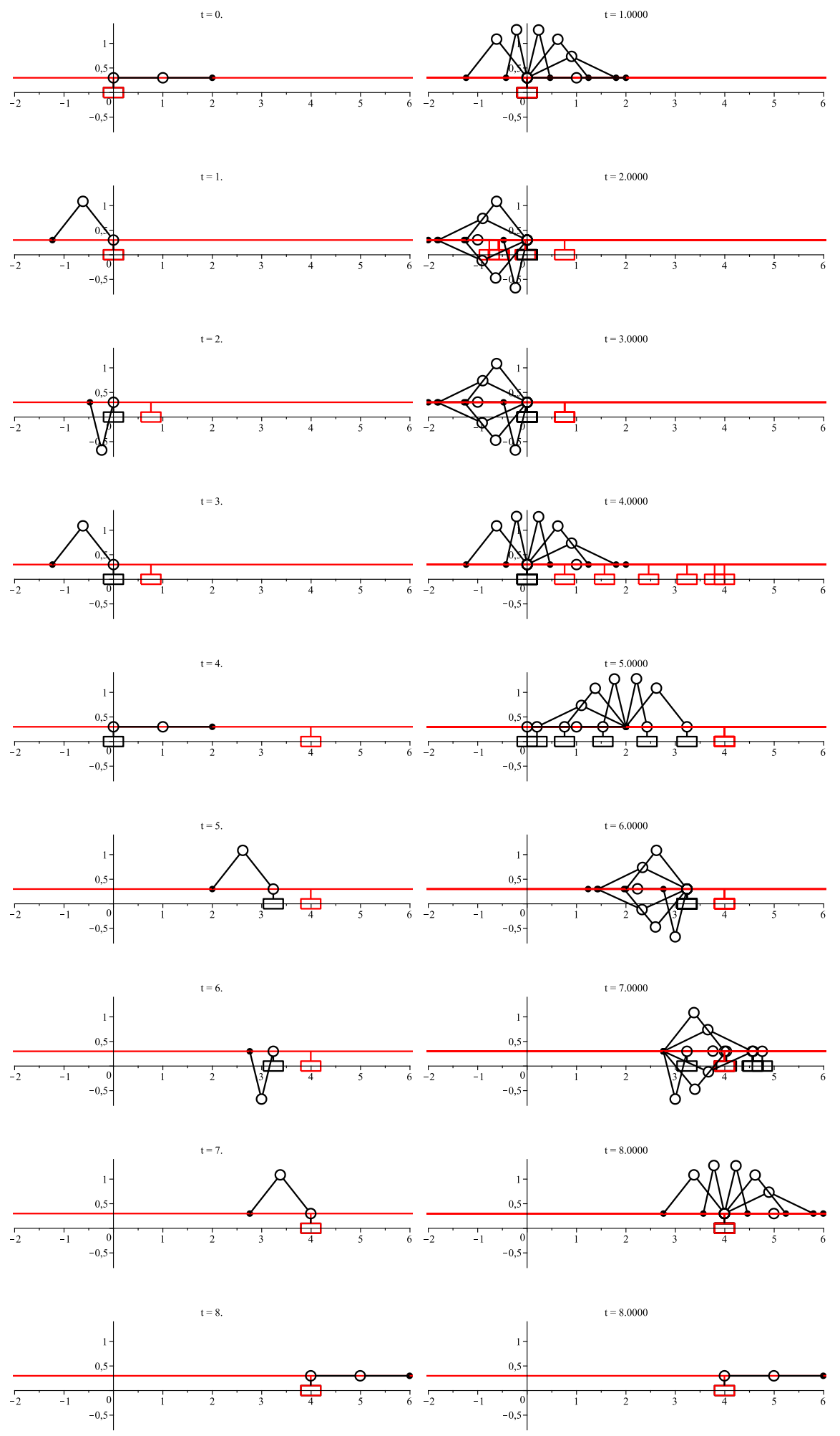

Fig. 18 Solution of the trajectory planning for the $R_{3}$ system. The sliding of the black rectangle and the red rectangle along the horizontal axis illustrate respectively the locomotion and the manipulation components of the motion. The first column displays snapshots of the motion taken at times of change of control fields (points where the curve in Fig. 17 changes color). The second column represents the transition motions between two successive snapshots. 


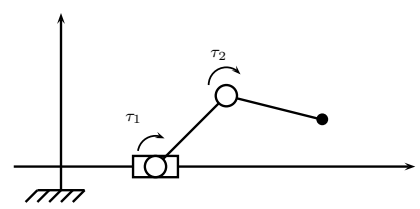

(a) Free mode, stratum $\mathcal{S}_{0}$

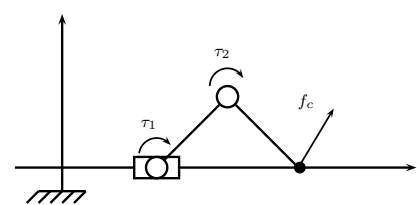

(b) Contact mode, stratum $\mathcal{S}_{1}$

Fig. 19 Forces and torques in the two modes

The efforts applied on $R_{1}$ in each of the two strata representing the two contact modes are portrayed in Fig. 19.

Using the Lagrangian approach, the dynamics of the system can be written as

$$
M(q) \ddot{q}+C(q, \dot{q}) \dot{q}+N(q, \dot{q})-J(q)^{T} f_{c}=\left(\begin{array}{c}
0 \\
\tau_{1} \\
\tau_{2}
\end{array}\right)
$$

which is in $\mathcal{S}_{1}$ (contact mode) when $f_{c} \neq 0$ and in $\mathcal{S}_{0}$ (free mode) when $f_{c}=0$; $M, C, N, J$ denote respectively the inertia matrix, the Coriolis and centrifugal effects, the external efforts (gravity, joint friction) vector, and the Jacobian matrix of the robot.

In the free mode, we can notice that $\ddot{\xi} \neq 0$ provided that the inertial effects of moving the links cause a dynamic reaction on the base. In the following we consider these links dynamics effects as perturbations and neglect them, which means that on the free mode $\ddot{\xi}=0^{3}$.

Let us now focus on the contact mode, which is our main concern in this study; $f_{c}$ is the Lagrange multiplier associated with the Lagrangian model of the system under the Pfaffian constraint $J(q) \dot{q}=0$. Solving the dynamic and the Pfaffian constraint equations for $f_{c}$ and $\ddot{q}$ leads

$$
f_{c}=-\left(J M^{-1} J^{T}\right)^{-1}\left(J M^{-1}(\tau-C \dot{q}-N)+\dot{J} \dot{q}\right)
$$

where $\tau=\left(0, \tau_{1}, \tau_{2}\right)^{T}$.

To avoid sliding, $f_{c}$ has to lie within the Coulomb friction cone $\mathcal{F}$ :

$$
f_{c} \in \mathcal{F}
$$

and

$$
\mathcal{F}=\left\{\left(f_{x}, f_{y}\right) \in \mathbb{R}^{2} \mid f_{y} \geq 0 \text { and }\left|f_{x}\right| \leq \mu f_{y}\right\}
$$

Now, we derive an open-loop control law $t \mapsto\left(\tau_{1}(t), \tau_{2}(t)\right)$ which steers the system from an initial contact state $\left(q_{i}, \dot{q}_{i}\right)$ to a final state $\left(q_{f}, \dot{q}_{f}\right)$ maintaining a non-sliding contact with the ground. To do so we adapt some of the ideas that were introduced in [42].

\footnotetext{
3 This is an ideal pure mathematical assumption that amounts to considering a relative inertia of the base link much bigger than that of the the two links. However, considering such an assumption on a real physical system implementing the model R3 may have severe effects on the system dynamics. In that case a separate control loop (a stabilizer module) can be provided for ensuring that $\xi=$ constant in the free mode without affecting the physical consistency and stability of the system.
} 
To make the derivations easier we neglect the masses of the links and consider only the mass of the sliding base $m_{0}{ }^{4}$. The dynamics equations become:

$$
\left\{\begin{array}{l}
m_{0} \ddot{\xi}=f_{x} \\
f_{x} \cdot\left(\sin \left(\theta_{1}\right)+l \sin \left(\theta_{1}+\theta_{2}\right)\right)-f_{y} \cdot\left(\cos \left(\theta_{1}\right)+\cos \left(\theta_{1}+\theta_{2}\right)\right)=\tau_{1} / l \\
f_{x} \cdot \sin \left(\theta_{1}+\theta_{2}\right)-f_{y} \cdot \cos \left(\theta_{1}+\theta_{2}\right)=\tau_{2} / l
\end{array}\right.
$$

In a given contact mode, the system evolves in a one-dimensional submanifold of the configuration space, a leaf of the stratum $\mathcal{S}_{1}$, that we parametrize with $\xi$. For example, if the contact is fixed at the abscissa 0 then $\xi=-2 l \cos \left(\theta_{1}\right)$ and $\theta_{2}=-2 \theta_{1}$. Solving equation (93) for $f_{x}$ and $f_{y}$ gives us

$$
\left\{\begin{array}{l}
f_{x}=\frac{\tau_{1}-2 \tau_{2}}{\sqrt{4 l^{2}-\xi^{2}}} \\
f_{y}=\frac{\tau_{1}}{\xi}
\end{array}\right.
$$

and the friction cone condition $f_{c} \in \mathcal{F}$, together with the maximum torques conditions $\left|\tau_{1}\right| \leq \tau_{\max }$ and $\left|\tau_{2}\right| \leq \tau_{\max }$ yields the following torque cone condition

$$
A_{\xi}\left(\begin{array}{c}
\tau_{1} \\
\tau_{2}
\end{array}\right) \leq b_{\xi}
$$

where

$$
A_{\xi}=\left(\begin{array}{cc}
\frac{1}{\sqrt{4 l^{2}-\xi^{2}}}-\frac{\mu}{\xi} & -\frac{2}{\sqrt{4 l^{2}-\xi^{2}}} \\
-\frac{1}{\sqrt{4 l^{2}-\xi^{2}}}-\frac{\mu}{\xi} & \frac{2}{\sqrt{4 l^{2}-\xi^{2}}} \\
-1 & 0 \\
1 & 0 \\
0 & -1 \\
0 & 1
\end{array}\right)
$$

and

$$
b_{\xi}=\left\{\begin{array}{l}
\left(0,0,0, \tau_{\max }, \tau_{\max }, \tau_{\max }\right)^{T}, \xi>0 \\
\left(0,0, \tau_{\max }, 0, \tau_{\max }, \tau_{\max }\right)^{T}, \xi<0
\end{array}\right.
$$

Finally, the open-loop dynamic trajectory planning reduces to

$$
\begin{aligned}
& \ddot{\xi}=f\left(\xi, \tau_{1}, \tau_{2}\right)=\frac{\tau_{1}-2 \tau_{2}}{m_{0} \sqrt{4 l^{2}-\xi^{2}}} \\
& \quad \text { under the constraint } A_{\xi}\left(\begin{array}{c}
\tau_{1} \\
\tau_{2}
\end{array}\right) \leq b_{\xi}
\end{aligned}
$$

or, putting $C_{\xi}$ the line matrix $C_{\xi}=\frac{1}{m_{0} \sqrt{4 l^{2}-\xi^{2}}}(1-2)$,

$$
\ddot{\xi}=C_{\xi}\left(\begin{array}{c}
\tau_{1} \\
\tau_{2}
\end{array}\right) \text { under the constraint } A_{\xi}\left(\begin{array}{c}
\tau_{1} \\
\tau_{2}
\end{array}\right) \leq b_{\xi}
$$

projecting in the dynamics and the constraints onto the space of task freedom as the term is defined in [42], using the change of control input $u=C_{\xi}\left(\begin{array}{l}\tau_{1} \\ \tau_{2}\end{array}\right)$, we get the simple double integrator $\ddot{\xi}=u$ where the torque cone condition translates

\footnotetext{
${ }^{4}$ Same remark as footnote ${ }^{3}$ above.
} 
into bounds on acceleration $u_{\min }(\xi) \leq u \leq u_{\max }(\xi)$. The time-optimal solution for this problem is known as the "bang-bang" control law [7], which consists in applying maximal acceleration forward from the initial state, maximal deceleration (i.e. minimal acceleration) backward from the final state, and switching between those two commands at the intersection point of the two trajectories obtained, see Fig. 20.

\subsection{Manipulation robot}

Similarly to $R_{1}$, we now consider the robot $R_{2}$ in the contact stratum $\mathcal{S}_{1}$. Let $m_{0}$ denote the mass of the sliding platform.

Let $f_{c}=\left(f_{x}, f_{y}\right) \in \mathbb{R}^{2}$ be the contact force applied by the sliding platform on the end-effector of the manipulator.

Instead of writing Newton's second law of motion applied to the platform and concatenating it with the Lagrangian dynamics of the manipulator coupled with the non-sliding contact point kinematic condition, we exhibit the equivalence with $R_{1}$ by directly writing the constrained Lagrangian problem for the whole system defined by its generalized coordinates $q=\left(\alpha, \theta_{1}, \theta_{2}\right)^{T}$. We get a similar equation to (89) where $f_{c}$ must be seen as the Lagrangian multiplier associated with $J(q) \dot{q}=0$, that we derived by differentiating the holonomic contact constraint

$$
h(q)=\alpha-2 \cos \left(\theta_{1}\right)+\text { constant }=0
$$

Thus, the following derivations follow the exact same scheme as for $R_{1}$, and we can control the variable $\alpha$ using the bang-bang control law.

\section{$5.3 \mathrm{~L} \& \mathrm{M}$ robot}

The dynamics and control of $R_{3}$ with the switching states control strategy considered in Section 3 simply reduce to the dynamics and control of $R_{1}$ and $R_{2}$ separately in each of the states of the system, respectively the locomotion and manipulation mode.

Instead, here we derive the dynamics of $R_{3}$ in contact mode (stratum $\mathcal{S}_{1}$ ) without the switching control input strategy. This can be seen as the dynamics of motion realized by taking a contact support on a mobile piece of the environment by the robot $R_{1}$, performing manipulation and locomotion at the same time.

Once again, instead of writing separately the dynamics of the subsystems made of the locomotor/manipulator and coupling them with the non-sliding contact point kinematic constraint, we directly consider the whole system $q=\left(\xi, \theta_{1}, \theta_{2}, \alpha\right)^{T}$ and write its Lagrangian equation of motion under the Pfaffian constraint which derives from the holonomic constraint

$$
h(q)=\left(\begin{array}{c}
\xi+l \cos \left(\theta_{1}\right)+l \cos \left(\theta_{1}+\theta_{2}\right)-\alpha+\text { constant } \\
l \sin \left(\theta_{1}\right)+l \sin \left(\theta_{1}+\theta_{2}\right)=0
\end{array}\right)=0
$$

So we get the following equation

$$
M(q) \ddot{q}+C(q, \dot{q}) \dot{q}+N(q, \dot{q})-J(q)^{T} f_{c}=\left(\begin{array}{c}
0 \\
\tau_{1} \\
\tau_{2} \\
0
\end{array}\right)
$$




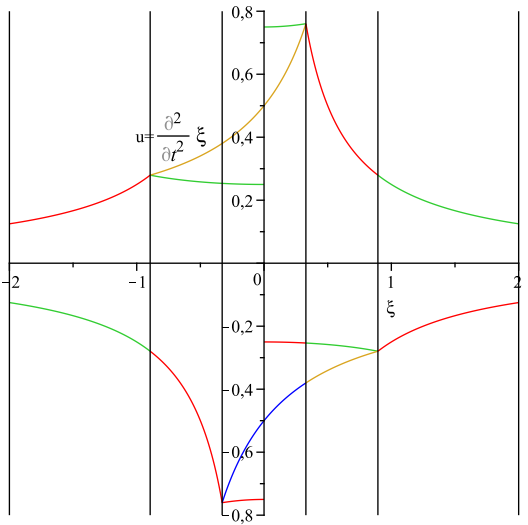

(a) Projection of torque cone in the $\ddot{\xi}$ space

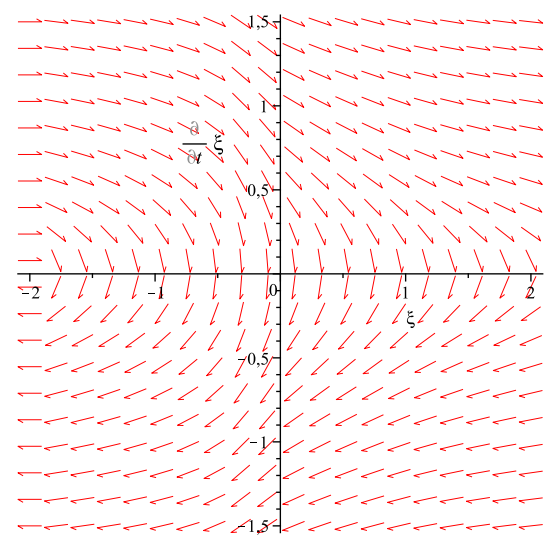

(c) Control field associated with $u_{\text {min }}$

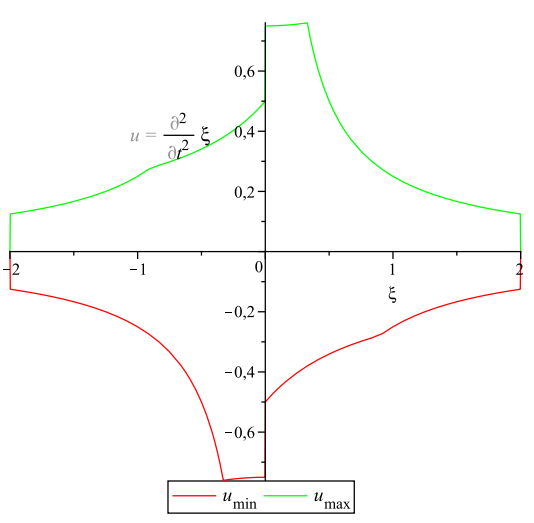

(b) Maximum acceleration and deceleration

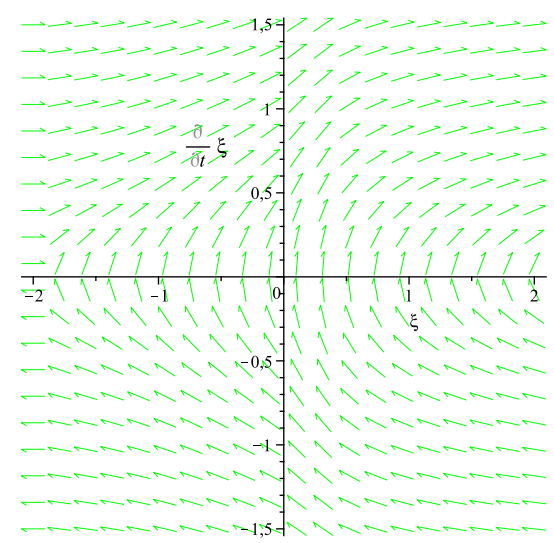

(d) Control field associated with $u_{\max }$

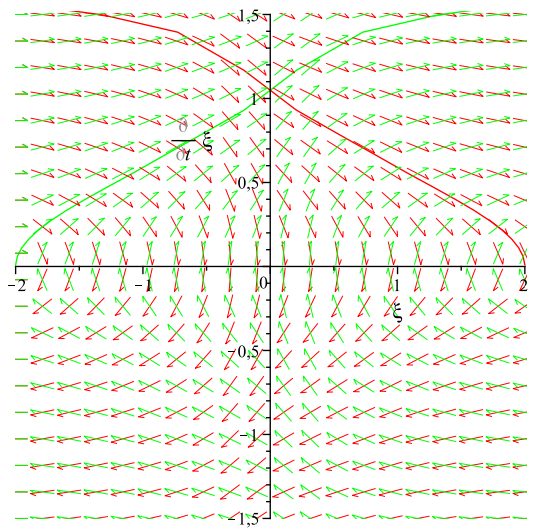

(e) Numerical integration and final solution

Fig. 20 Bang-bang control law synthesis 
Table 1 Parameter values for the simulations

\begin{tabular}{ccc}
\hline Parameter & value & unit \\
\hline$l$ & 1 & $\mathrm{~m}$ \\
$m_{0}$ & 2 & $\mathrm{~kg}$ \\
$m_{1}$ & 0 & $\mathrm{~kg}$ \\
$m_{2}$ & 0 & $\mathrm{~kg}$ \\
$\tau_{\max }$ & 1 & $\mathrm{~N} . \mathrm{m}$ \\
$\mu$ & $1 / 2$ & - \\
$m_{\xi}$ & 8 & $\mathrm{~kg}$ \\
$m_{\alpha}$ & 4 & $\mathrm{~kg}$ \\
\hline
\end{tabular}

Let us now generate a control law for $R_{3}$ in contact mode under Coulomb friction hypothesis. For the sake of clarity and without loss of generality we suppose that the end effector is fixed at the location $\beta=0$. Neglecting the masses of the link gives us the following dynamics equation:

$$
\left(\begin{array}{cccc}
m_{\xi} & 0 & \cdots & 0 \\
0 & 0 & \ddots & \vdots \\
\vdots & \ddots & 0 & 0 \\
0 & \cdots & 0 & m_{\alpha}
\end{array}\right)\left(\begin{array}{c}
\ddot{\xi} \\
\ddot{\theta}_{1} \\
\ddot{\theta}_{2} \\
\ddot{\alpha}
\end{array}\right)+\left(\begin{array}{cc}
1 & 0 \\
0 & \alpha-\xi \\
\sqrt{1-\left(\frac{\alpha-\xi}{2}\right)^{2}} & \frac{\alpha-\xi}{2} \\
-1 & 0
\end{array}\right)\left(\begin{array}{c}
f_{x} \\
f_{y}
\end{array}\right)=\left(\begin{array}{c}
0 \\
\tau_{1} \\
\tau_{2} \\
0
\end{array}\right)
$$

If we introduce the new variable $\delta=\xi-\alpha$ which expresses the displacement of the locomotor in the platform's inertial frame, we can rewrite the equation as

$$
\left\{\begin{array}{l}
\frac{m_{\xi}-m_{\alpha}}{2} \ddot{\delta}+f_{x}=0 \\
-\delta f_{y}=\tau_{1} \\
\sqrt{1-\frac{\delta^{2}}{4}} f_{x}-\frac{\delta}{2} f_{y}=\tau_{2}
\end{array}\right.
$$

which are the same equations as the ones we got respectively for $\xi$ and $\alpha$ in $R_{1}$ and $R_{2}$, with a virtual mass $m_{0}=\frac{m_{\xi}-m_{\alpha}}{2}$. We can thus control the variable $\delta$ with the same control law as in Section 5.1 which is not any more valid for $\xi$ and $\alpha$ separately, that is, the bang-bang control law for the double integrator $\ddot{\delta}=u$ with the solution $t \mapsto u(t)$ depicted in Fig. 20 .

Table 1 provides the numerical values of the parameters used in the simulations throughout the paper.

\section{Discussion}

\subsection{Perspectives for high-dimensional systems}

Our work paves the way towards addressing high-dimensional systems, from both a conceptual and a methodological point-of-view.

Indeed, this study gives a precise definition and formulation to the desired paradigm of non-decoupled locomotion-and-manipulation planning and control, and what we mean exactly by that terminology. It concretely illustrates what kind of results we would be expecting from the implementation of such a paradigm. The expected form of results (locomotion-while-manipulation) readily extends to 
higher-dimensional systems. Hence we know what kind of motion to aim for when considering the extension to the latter systems.

More specifically, we can see that the achieved results are conceptually distinct from the existing ones coordinating locomotion and manipulation, where the robot uses locomotion to move to a spot that enables manipulation $[28,29,17,39,46]$. Instead, we proposed a novel locomotion-and-manipulation paradigm that consists in 1) specifying a task to the locomotion-and-manipulation system in the form of only the final position/configuration of the robot and/or the manipulated object, and 2) letting the planner and the controller figure out autonomously by themselves how to decompose the motion in as many pure locomotion, pure manipulation, or locomotion-while-manipulation elementary motion fragments as necessary to realize the task, solving implicitly the underactuation of the system. The decomposition happens at whichever instants deemed necessary in the overall motion. It does not restrict to a locomotion block followed by a manipulation block or viceversa. We successfully illustrated the paradigm and we exhibited what class of resulting locomotion-while-manipulation motion emerges from it, e.g. the motion illustrated in Fig. 18.

Armed with that particular paradigm instantiation, that we proved was analytically tractable in minimal systems, it becomes possible to directly specify the same requirements and formulation (the same paradigm) for higher dimensional systems such as humanoids. For those however, we need, in a first approach, to use alternative solutions that are not exact solutions or closed-form solutions as presented in this work. We rather resort to more heuristics-based ones, due to the general non-tractability of the analytical methods in high-dimensional spaces.

Hence, following the same logic that we detailed in this paper, but using alternative particular methods of resolution based on heuristics, we were able to design a non-decoupled planning algorithm that answers the same paradigm for locomotion, manipulation, or locomotion-while-manipulation of high-dimensional humanoid systems in [10] (building on a high-dimensional posture generator written as non-linear optimization problem $[8,9,37]$ and a general dynamics algorithm for high-dimensional systems [11]). The algorithm is applicable to even higherdimensional systems by considering systems made of two or more humanoid robots collaborating to manipulate objects or mechanisms. We followed that planner with a dynamics controller that is able to realize high-level locomotion, manipulation, or locomotion-while-manipulation tasks in a dynamically consistent way in [43]. This is a first step towards fully extending the methods presented in this paper to the high-dimensional systems. Other strategies for dimensionality reduction of the high-dimensional systems of this framework have been explored in $[12,13]$.

As an example, Fig. 21 illustrates a resulting motion of the paradigm introduced in this paper for a humanoid robot, as instantiated in the works $[10,43]$ higher-dimensional systems. The objective in Fig. 21 is specified as a final position for the humanoid robot ( 2 meters forward) and a final orientation of the manipulated box (purple face facing upwards). Each frame of the motion has to be physically consistent (equation of dynamics satisfied for both the robot and the box, forces applied by the robot on the box opposite to the forces applied by the box on the robot, contact forces inside the friction cones, etc.). The motion planner figures out by itself how to manipulate the box while walking, fitting in the paradigm we defined in the analytical case. 
Furthermore, results from the present works can be used to assess theoretical consistency of the latter high-dimensional heuristics. As a concrete example for this, take the generalization of Corollary 1, which can be reached in future work for example by a recursive reasoning to go from a configuration space of dimension $n$ to a configuration space of dimension $n-1$. The generalization can be used to theoretically prove the completeness of the high-dimensional heuristics-based algorithm of [10], i.e. to formally prove that the algorithm find a solution whenever one exists when using randomized (RRT, PRM) planners that do not account for the stratification/foliation of the configuration space. This is another example of a step towards the extension of the present work to the high-dimensional systems as future work.

\subsection{Limitations}

The R3 robot uses the same means of locomotion and manipulation, which results in autonomously decomposing the motion in elementary motion fragments that consist in either pure locomotion fragment, pure manipulation fragment, or pure contact repositioning fragment. However, by the remark of Section 3, and by Corollary 1, either the pure locomotion fragments or pure manipulation fragments can be replaced with locomotion-while-manipulation elementary fragments by setting an arbitrary value of $\left(\lambda_{1}, \lambda_{2}\right)=\left(\lambda_{1}, 1-\lambda_{1}\right)$ and using the corresponding $\left(\lambda_{1}, \lambda_{2}\right)$-foliation of the bottom stratum, instead of either the $\alpha$-foliation or the $\xi$-foliation. This remark extends also to the control part of Section 4.3 in which one of the vector fields $g_{2}$ or $g_{3}$ can be replaced with a linear combination $\lambda_{1} g_{2}+\lambda_{2} g_{3}$ which would result in elementary motion fragments in which both the base of the manipulator and the manipulated object translate simultaneously.

Yet, One of the assumption (and limitation) of the approach presented in section 4 is that the solutions consist in following the vector fields sequentially and never a linear combination of these vector fields. Hence, for systems in which the locomotion and manipulation mechanical components are decoupled, this would result in non-optimal motions. In that case, a parallelization of the kinematic model of the system could be implemented to optimally use the mechanical ressources of the system. For example, one possible approach is to decompose the system in elementary minimal locomotion, manipulation, or locomotion-and-manipulation systems in a complementary way and to implement the presented framework in parallel on each of these subsystems. Such an automatic decomposition of the kinematic model, parallelization, and framework adaptation can be the subject of a future study.

\section{Conclusion}

The motion planning and control problems for the systems $R_{1}$ (locomotion-only), $R_{2}$ (manipulation-only), and $R_{3}$ (simultaneous locomotion and manipulation) are solvable with the same tools. We proved the reduction property for all three systems. This property reduces the path planning problem in a foliated configuration space to a classical path planning problem in a non-foliated space. The formulation of the motion planning problem as a BVP was written for the three systems, and 
non-holonomic trajectory planning techniques were used for solving this problem. Dynamics derivations also appeared to be equivalent for the three robots. The Lagrangian approach acting on one global system with generalized coordinates instead of different subsystems as traditionally considered turned out to be a powerful unification tool for making these derivations.

We thus successfully applied a set of three motion planning methods for the three systems $R_{1}, R_{2}$, and $R_{3}$. By doing so, we showed that our initial paradigm, the simultaneous non-decoupled locomotion and manipulation planning, holds using any of these methods.

Though being theory-oriented, our study was primarily motivated by the practical humanoid robot motion planning issues, given that a humanoid robot is a platform with both locomotion and manipulation capabilities. The targeted objective was to integrate and fuse works done on humanoid locomotion planning $[18,23]$ and dexterous manipulation planning $[48,40,45]$, or any other type of whole-body manipulation planning $[47,20]$, within one non-decoupled planning framework. In this work, we precisely defined the desired paradigm of locomotion-while-manipulation and we laid down the theoretical foundations that would justify the same paradigm in higher-dimensional locomotion-while-manipulation systems such as humanoids. We achieved that in $[10,43]$, using an alternative instantiation of the paradigm more adapted to high-dimensional spaces, and more based on heuristics. The next step of the work will be to generalize some of the fundamental results of this paper (e.g. Corollary 1) to prove the completeness of the algorithms in e.g. [10].

\section{Acknowledgment}

This work was partially supported by the H2020 COMANOID EU project www . comanoid. eu and the by the JSPS Grant-in-Aid for Scientific Research (B) Number 16H02886 ("Cutting-Edge multi-contact behaviors").

\section{References}

1. Alami, R., Laumond, J.P., Siméon, T.: Two Manipulation Planning Algorithms. In: Proceedings of the Workshop on Algorithmic Foundations of Robotics, pp. 109-125 (1995)

2. Ames, A.D., Powell, M.: Towards the unification of locomotion and manipulation through control lyapunov functions and quadratic programs. In: Control of Cyber-Physical Systems, pp. 219-240. Springer (2013)

3. Arai, T.: Robots with integrated locomotion and manipulation and their future. In: Intelligent Robots and Systems' 96, IROS 96, Proceedings of the 1996 IEEE/RSJ International Conference on, vol. 2, pp. 541-545. IEEE (1996)

4. Avnaim, F., Boissonnat, J.D.: Polygon Placement Under Translation and Rotation. In: Symposium on Theoretical Aspects of Computer Science, pp. 322-333 (1988)

5. Ben-Tzvi, P.: Hybrid Mobile Robot Systems: Interchanging Locomotion and Manipulation. Ph.D. thesis, University of Toronto (2008)

6. Ben-Tzvi, P., Goldenberg, A., Zu, J.: Design and Analysis of a Hybrid Mobile Robot Mechanism with Compounded Locomotion and Manipulation Capability. Transactions of the ASME Journal of Mechanical Design 130, 1-13 (2008)

7. Bobrow, J., Dubowsky, S., Gibson, J.: Time-Optimal Control of Robotic Manipulators Along Specified Paths. International Journal of Robotics Research 4(3), 3-17 (1985)

8. Bouyarmane, K., Kheddar, A.: Static multi-contact inverse problem for multiple humanoid robots and manipulated objects. In: IEEE-RAS International Conference on Humanoid Robots, pp. 8-13 (2010) 
9. Bouyarmane, K., Kheddar, A.: Fem-based static posture planning for a humanoid robot on deformable contact support. In: IEEE-RAS International Conference on Humanoid Robots, pp. 487-492 (2011)

10. Bouyarmane, K., Kheddar, A.: Humanoid robot locomotion and manipulation step planning. Advanced Robotics 26(10), 1099-1126 (2012)

11. Bouyarmane, K., Kheddar, A.: On the dynamics modeling of free-floating-base articulated mechanisms and applications to humanoid whole-body dynamics and control. In: IEEERAS International Conference on Humanoid Robots (Humanoids 2012), pp. 36-42 (2012)

12. Bouyarmane, K., Vaillant, J., Morimoto, J.: Low-dimensional user control of autonomously planned whole-body humanoid locomotion motion towards brain-computer interface applications, pp. 740-748. DOI 10.1142/9789814525534_0092

13. Bouyarmane, K., Vaillant, J., Sugimoto, N., Keith, F., Furukawa, J.i., Morimoto, J.: Brainmachine interfacing control of whole-body humanoid motion. Frontiers in Systems Neuroscience 8, 138 (2014). DOI 10.3389/fnsys.2014.00138

14. Bullo, F., Lewis, A.D.: Geometric Control of Mechanical Systems. Springer (2000)

15. Choset, H., Lynch, K.M., Hutchinson, S., Kantor, G., Burgard, W., Kavraki, L.E., Thrun, S.: Principles of Robot Motion: Theory, Algorithms, and Implementations. The MIT Press (2005)

16. Craig, J.J.: Introduction to Robotics: Mechanics and Control (3rd Edition). Prentice Hall (2004)

17. Dang, D., Lamiraux, F., Laumond, J.P.: A framework for manipulation and locomotion with realtime footstep replanning. In: Humanoid Robots (Humanoids), 2011 11th IEEERAS International Conference on, pp. 676-681. IEEE (2011)

18. Escande, A., Kheddar, A., Miossec, S.: Planning support contact-points for humanoid robots and experiments on HRP-2. In: IEEE/RSJ International Conference on Intelligent Robots and Systems, pp. 2974-2979. Beijing, China (2006)

19. Escande, A., Kheddar, A., Miossec, S.: Planning contact points for humanoid robots. Robotics and Autonomous Systems 61(5), 428 - 442 (2013). DOI 10.1016/j.robot.2013. 01.008

20. Esteves, C., Arechavelata, G., Pettré, J., Laumond, J.P.: Animation Planning for Virtual Characters Cooperation. ACM Transactions on Graphics 25(2), 319-339 (2006)

21. Goodwine, B., Burdick, J.: Controllability of Kinematic Control Systems on Stratified Configuration Spaces. IEEE Transactions on Automatic Control 46(3), 358-368 (2001)

22. Goodwine, B., Burdick, J.: Motion Planning for Kinematic Stratified Systems With Application to Quasi-Static Legged Locomotion and Finger Gaiting. IEEE Transactions on Robotics and Automation 18(2), 209-222 (2002)

23. Hauser, K., Bretl, T., Latombe, J.C., Harada, K., Wilcox, B.: Motion Planning for legged Robots on Varied Terrain. International Journal of Robotics Research 27(11-12), 13251349 (2008)

24. Hauser, K., Ng-Thow-Hing, V., Gonzalez-Baños, H.: Multi-modal motion planning for a humanoid robot manipulation task. In: M. Kaneko, Y. Nakamura (eds.) International Symposium Robotics Research, pp. 307-317. Springer Berlin Heidelberg, Berlin, Heidelberg (2011)

25. Hopcroft, J., Wilfong, G.: Motion of Objects in Contact. International Journal of Robotics Research 4(4), 32-46 (1986)

26. Isham, C.: Modern Differential Geometry for Physicists, World Scientific Lecture Notes in Physics, vol. 61, 2nd edn. World Scientific (1999)

27. Kajita, S., Espiau, B.: Springer Handbook of Robotics, chap. Legged Robots. Springer (2008)

28. Kanoun, O., Laumond, J.: Optimizing the Stepping of a Humanoid Robot for a Sequence of Tasks. In: IEEE-RAS International Conference on Humanoid Robots (2010)

29. Kanoun, O., Laumond, J., Yoshida, E.: Planning Foot Placements for a Humanoid Robot : a Problem of Inverse Kinematics. International Journal of Robotics Research 30(4), 476-485 (2011)

30. Kemp, C.C., Fitzpatrick, P., Hirukawa, H., Yokoi, K., Harada, K., Matsumoto, Y.: Springer Handbook of Robotics, chap. Humanoids. Springer (2008)

31. Koyachi, N., Arai, T., Adachi, H., Itoh, Y.: Integrated limb mechanism of manipulation and locomotion for dismantling robot-basic concept for control and mechanism. In: Advanced Robotics, 1993. Can Robots Contribute to Preventing Environmental Deterioration? Proceedings, 1993 IEEE/Tsukuba International Workshop on, pp. 81-84. IEEE (1993) 
32. Lafferriere, G., Sussmann, H.J.: A Differential Geometric Approach to Motion Planning. In: Nonholonomic Motion Planning, pp. 235-270. Kluwer (1993)

33. Latombe, J.C.: Robot Motion Planning. Kluwer Academic Publishers (1991)

34. Laumond, J.P.: Robot Motion Planning and Control. Springer (1998)

35. LaValle, S.M.: Planning Algorithms. Cambridge University Press (2006)

36. M'closkey, R.T., Murray, R.M.: Exponential stabilization of driftless nonlinear control systems using homogeneous feedback. IEEE Transactions on automatic control 42(5), 614-628 (1997)

37. Moulard, T., Lamiraux, F., Bouyarmane, K., Yoshida, E.: Roboptim: an optimization framework for robotics. In: Robomec, p. 4 (2013)

38. Murray, R.M., Li, Z., Sastry, S.S.: A Mathematical Introduction to Robotic Manipulation. CRC Press (1994)

39. Saab, L., Soueres, P., Fourquet, J.: Coupling manipulation and locomotion tasks for a humanoid robot. In: Advances in Computational Tools for Engineering Applications, 2009. ACTEA'09. International Conference on, pp. 84-89. IEEE (2009)

40. Saut, J.P., Sahbani, A., El-Khoury, S., Perdereau, V.: Dexterous manipulation planning using probabilistic roadmaps in continuous grasp subspaces. In: IEEE/RSJ International Conference on Intelligent Robots and Systems, pp. 2907-2912. San Diego, CA, USA (2007)

41. Siméon, T., Laumond, J.P., Cortés, J., Sahbani, A.: Manipulation Planning With Probabilistic Roadmaps. International Journal of Robotics Research 23(7-8), 729-746 (2004)

42. Srinivasa, S.S., Erdmann, M.A., Mason, M.T.: Using Projected Dynamics to Plan Dynamic Contact Manipulation. In: IEEE/RSJ International Conference on Intelligent Robots and Systems, pp. 3618-3623. Edmonton, Alta., Canada (2005)

43. Vaillant, J., Bouyarmane, K., Kheddar, A.: Multi-character physical and behavioral interactions controller. IEEE Transactions on Visualization and Computer Graphics 23(6), 1650-1662 (2017)

44. Vaillant, J., Kheddar, A., Audren, H., Keith, F., Brossette, S., Escande, A., Bouyarmane, K., Kaneko, K., Morisawa, M., Gergondet, P., Yoshida, E., Kajita, S., Kanehiro, F.: Multicontact vertical ladder climbing with an HRP-2 humanoid. Autonomous Robots 40(3), 561-580 (2016). DOI 10.1007/s10514-016-9546-4

45. Xu, J., Koo, J., Li, Z.: Finger gaits planning for multifingered manipulation. In: IEEE/RSJ International Conference on Intelligent Robots and Systems, pp. 2932-2937. San Diego, CA, USA (2007)

46. Yamamoto, Y., Yun, X.: Coordinating locomotion and manipulation of a mobile manipulator. In: Decision and Control, 1992., Proceedings of the 31st IEEE Conference on, pp. 2643-2648. IEEE (1992)

47. Yamane, K., Kuffner, J., Hodgins, J.K.: Synthesizing animations of human manipulation tasks. ACM Transactions on Graphics (SIGGRAPH) 23(3) (2004)

48. Yashima, M., Shiina, Y., Yamaguchi, H.: Randomized manipulation planning for a multifingered hand by switching contact modes. In: IEEE International Conference on Robotics and Automation, vol. 2, pp. 2689-2694 (2003)

49. Yoshida, E., Esteves, C., Kanoun, O., Poirier, M., Mallet, A., Laumond, J.P., Yokoi, K.: Planning whole-body humanoid locomotion, reaching, and manipulation. In: Motion Planning for Humanoid Robots, pp. 99-128. Springer (2010) 


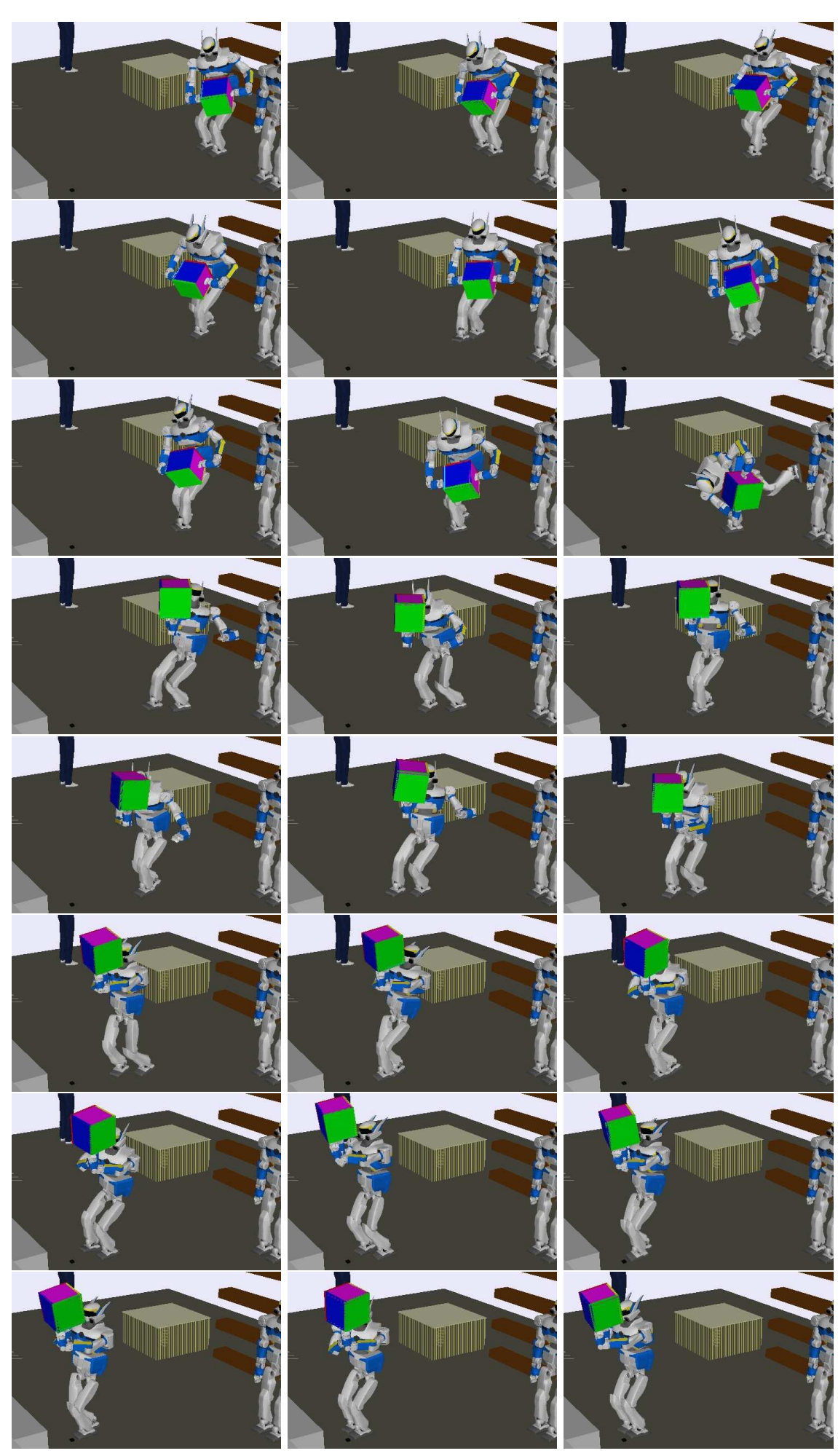

Fig. 21 The objective is for the HRP-2 robot to advance $2 \mathrm{~m}$ forward while simultaneously performing half rotation of the $5 \mathrm{~kg}$ box, bringing the purple face up. Friction coefficients between the hands and the box are set to $\mu=1$. The robot autonomously re-grasps and rotates the box while walking. 